\title{
Structure of Management Information for version 2 of the Simple Network Management Protocol (SNMPv2)
}

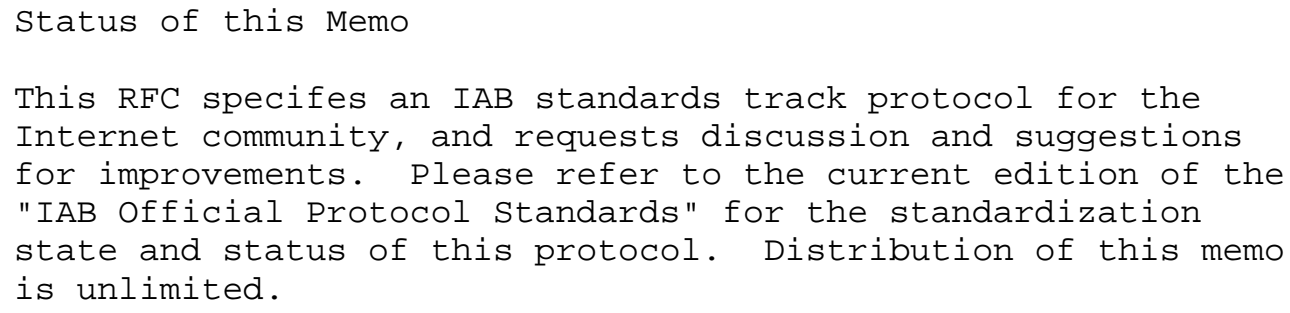

Table of Contents

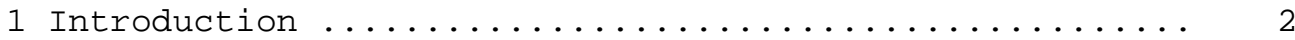

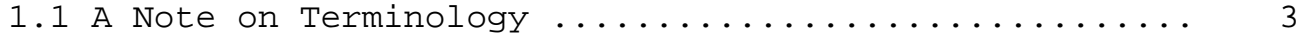

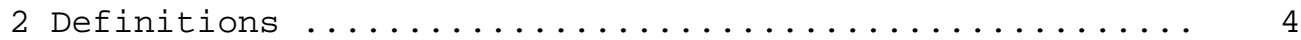

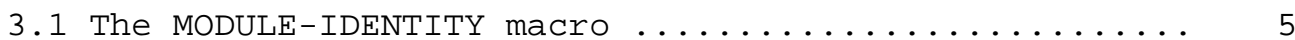

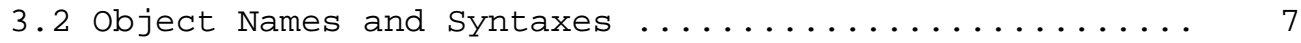

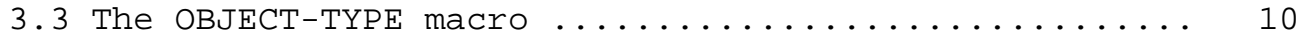

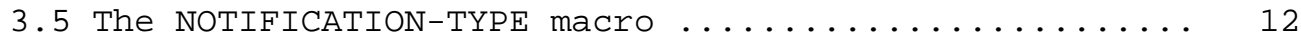

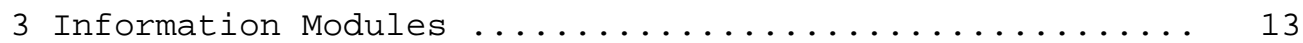

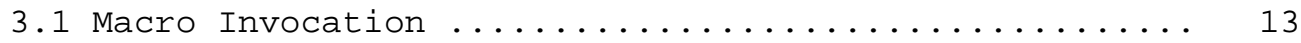

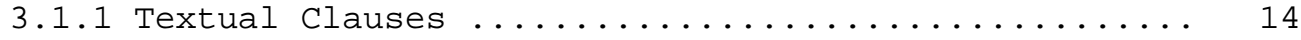

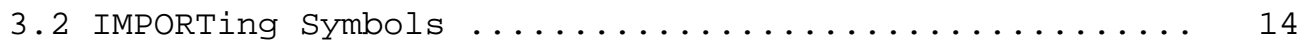

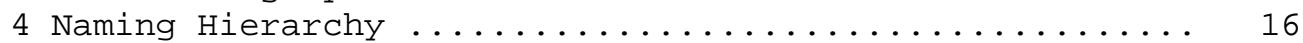

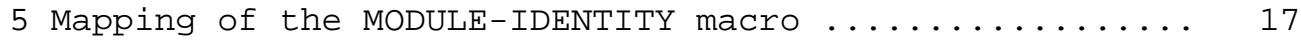

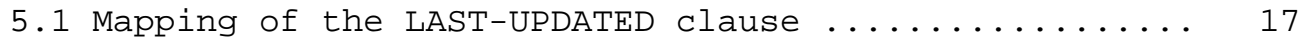

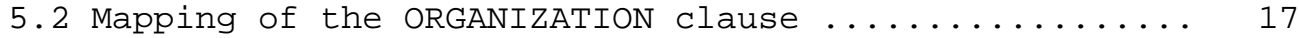

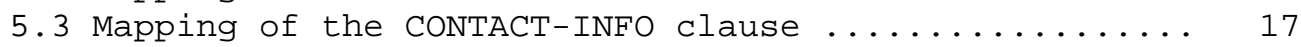

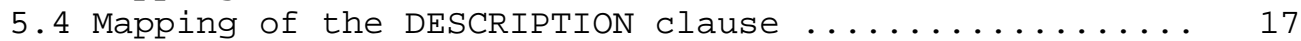

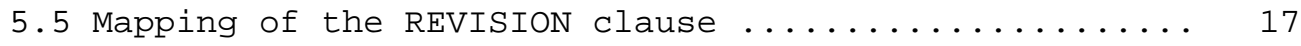

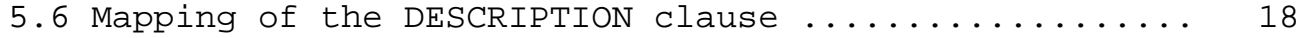

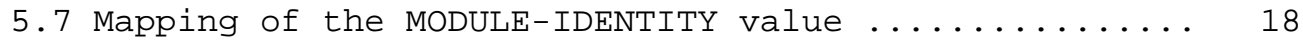

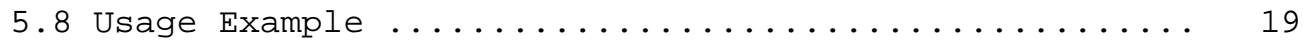

Case, McCloghrie, Rose \& Waldbusser [Page i] 


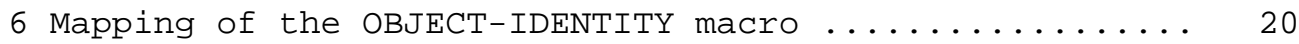

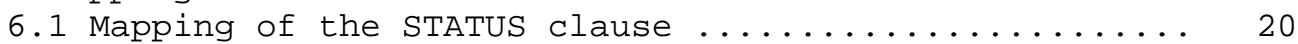

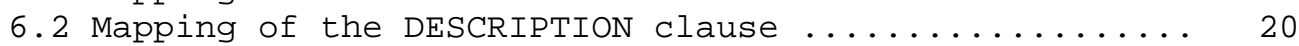

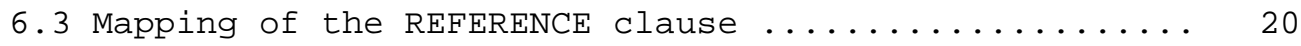

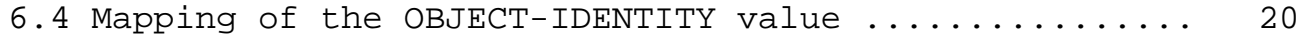

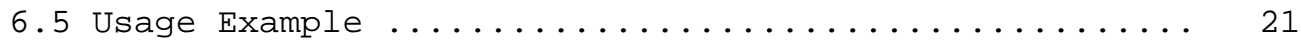

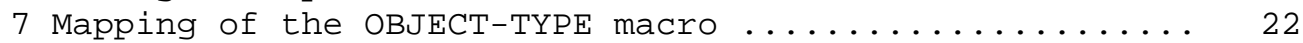

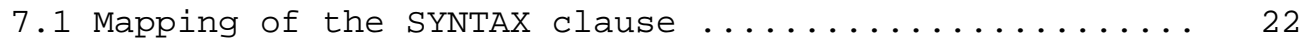

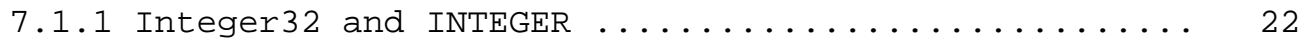

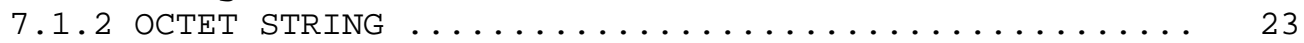

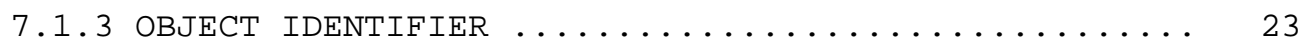

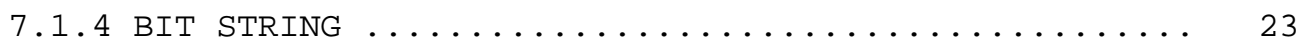

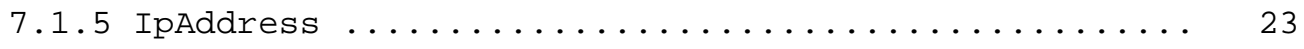

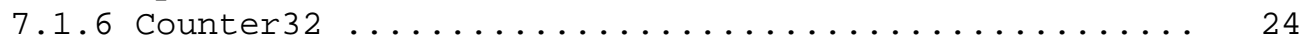

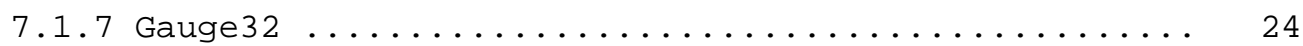

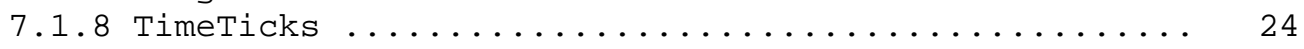

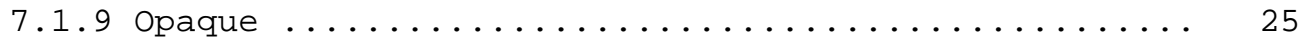

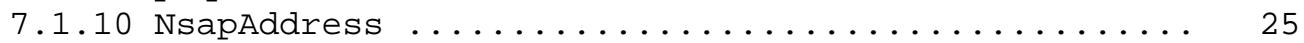

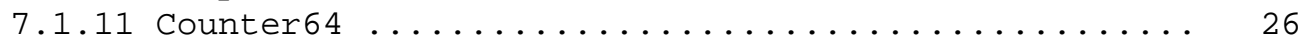

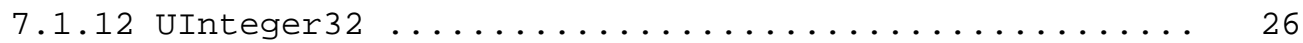

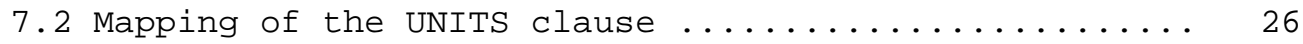

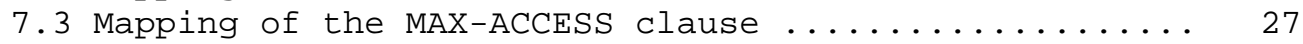

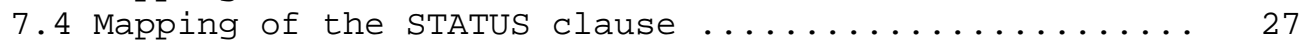

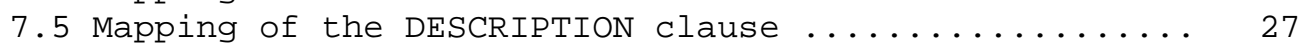

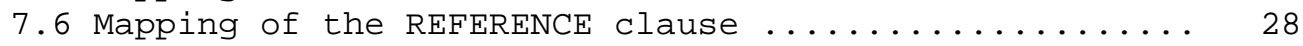

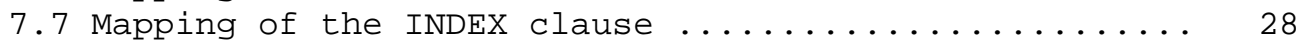

7.7.1 Creation and Deletion of Conceptual Rows ....... 30

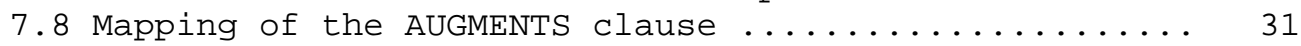

7.8.1 Relation between INDEX and AUGMENTS clauses ...... 31

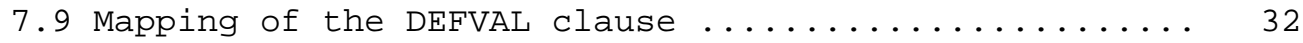

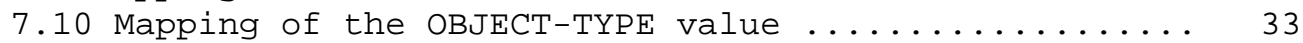

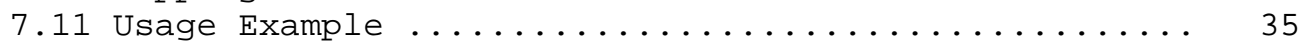

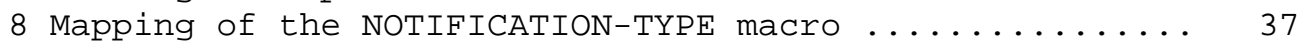

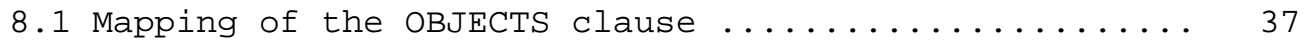

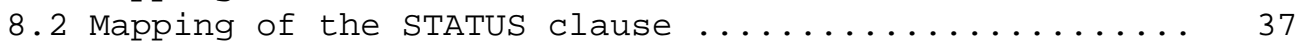

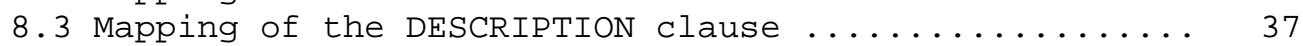

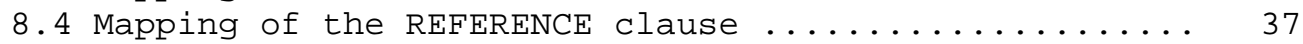

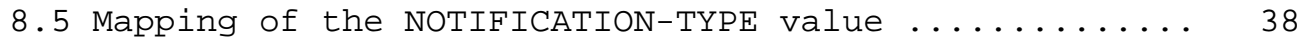

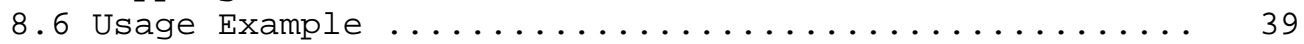

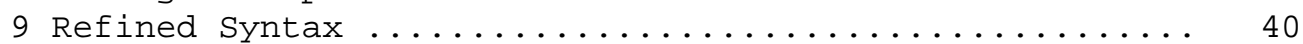

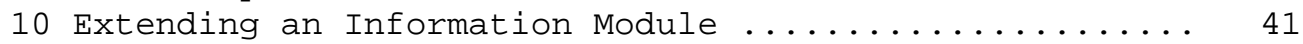

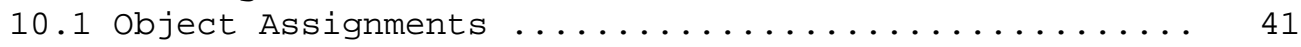

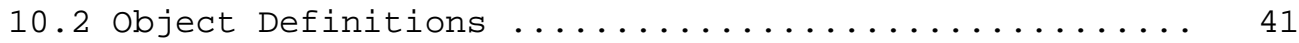

10.3 Notification Definitions .................. 42

Case, McCloghrie, Rose \& Waldbusser [Page ii] 
11 Appendix: de-OSIfying a MIB module .............. 43

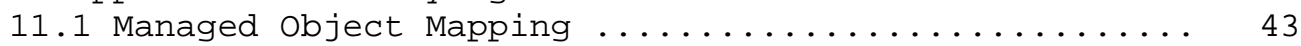

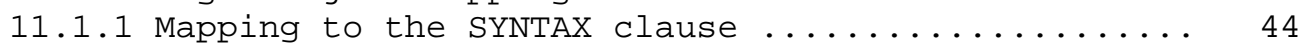

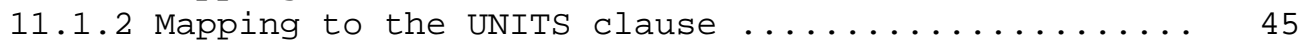

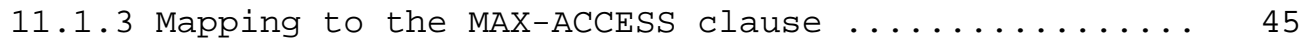

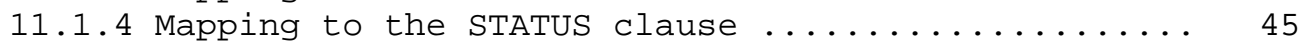

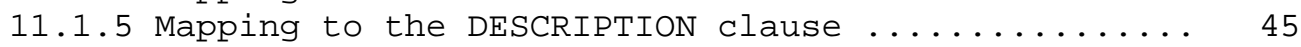

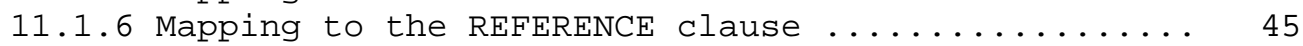

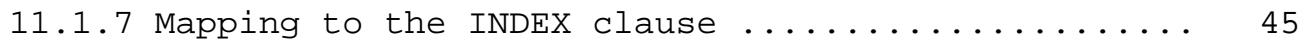

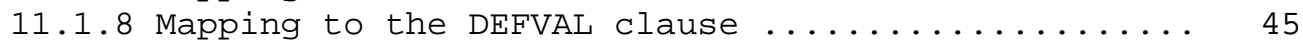

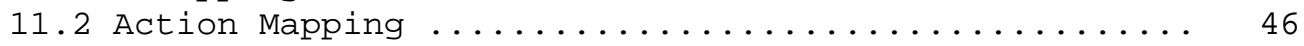

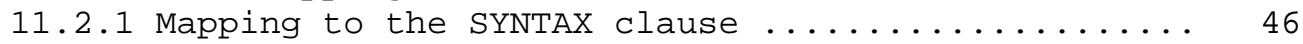

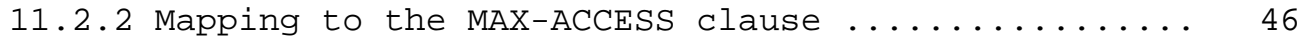

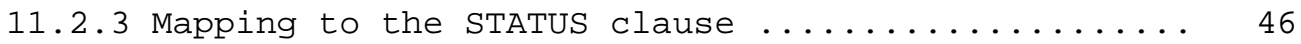

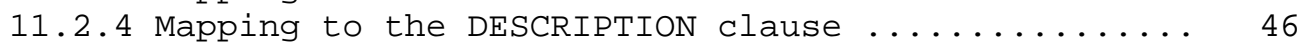

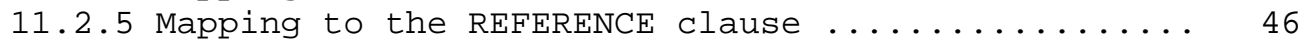

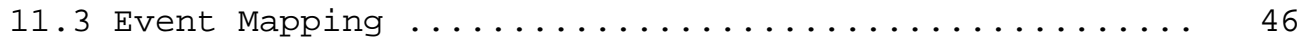

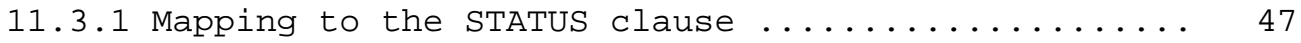

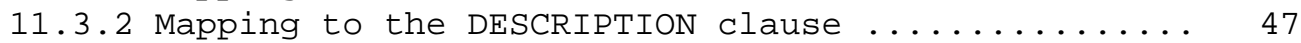

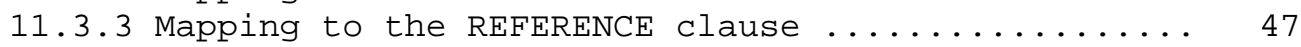

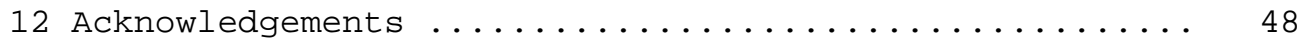

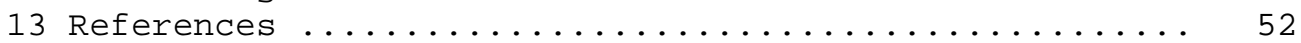

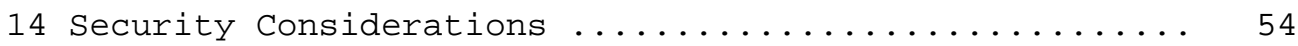

15 Authors' Addresses ........................ 54

Case, McCloghrie, Rose \& Waldbusser [Page 1] 


\section{Introduction}

A network management system contains: several (potentially many) nodes, each with a processing entity, termed an agent, which has access to management instrumentation; at least one management station; and, a management protocol, used to convey management information between the agents and management stations. Operations of the protocol are carried out under an administrative framework which defines both authentication and authorization policies.

Network management stations execute management applications which monitor and control network elements. Network elements are devices such as hosts, routers, terminal servers, etc., which are monitored and controlled through access to their management information.

Management information is viewed as a collection of managed objects, residing in a virtual information store, termed the Management Information Base (MIB). Collections of related objects are defined in MIB modules. These modules are written using a subset of OSI's Abstract syntax Notation One (ASN.1)

[1]. It is the purpose of this document, the structure of Management Information (SMI), to define that subset.

The SMI is divided into three parts: module definitions, object definitions, and, trap definitions.

(1) Module definitions are used when describing information modules. An ASN.1 macro, MODULE-IDENTITY, is used to concisely convey the semantics of an information module.

(2) Object definitions are used when describing managed objects. An ASN.1 macro, OBJECT-TYPE, is used to concisely convey the syntax and semantics of a managed object.

(3) Notification definitions are used when describing unsolicited transmissions of management information. An ASN.1 macro, NOTIFICATION-TYPE, is used to concisely convey the syntax and semantics of a notification. 
1.1. A Note on Terminology

For the purpose of exposition, the original Internet-standard Network Management Framework, as described in RFCs 1155, 1157, and 1212, is termed the SNMP version 1 framework (SNMPV1). The current framework is termed the SNMP version 2 framework (SNMPV2) . 


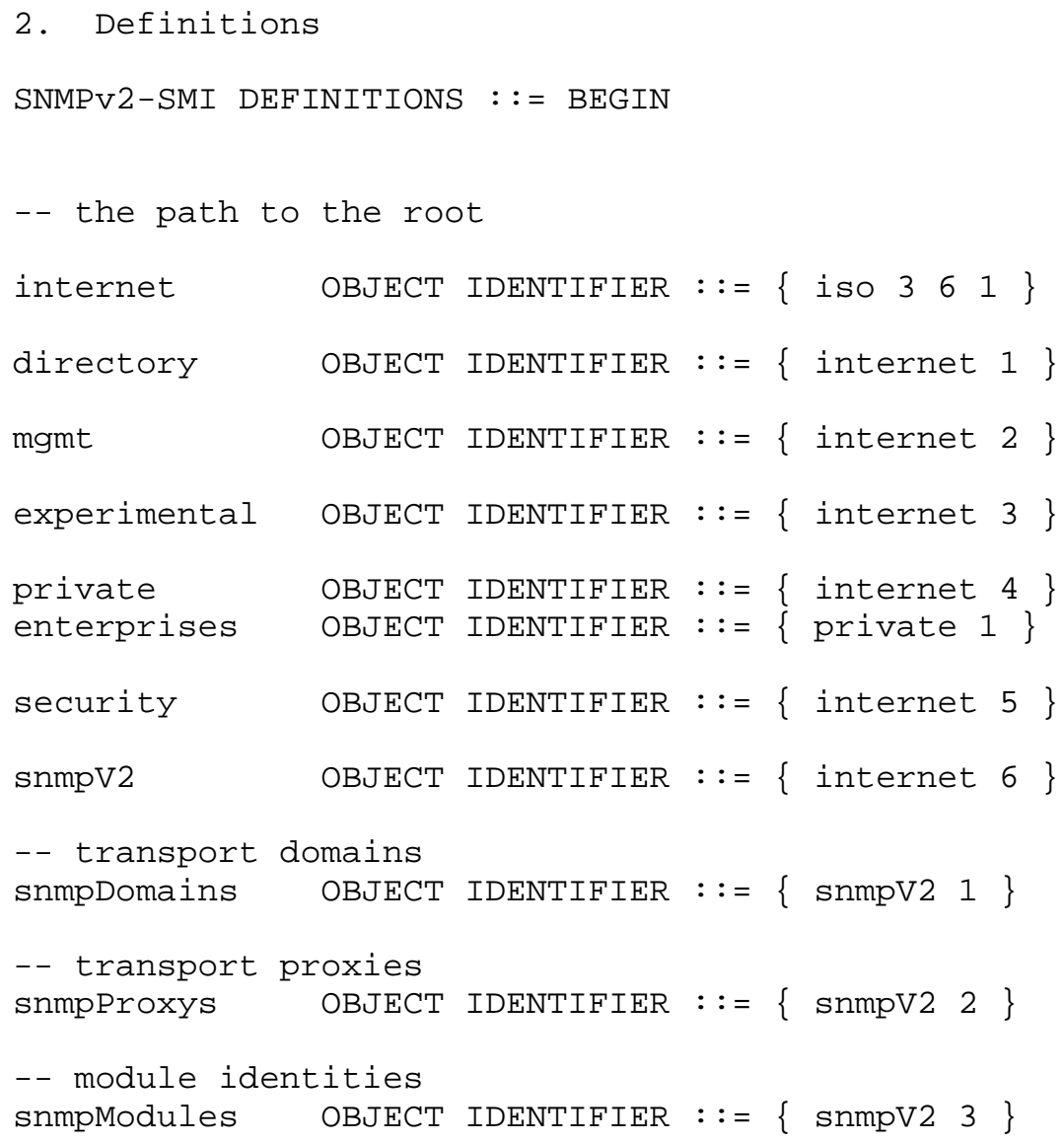




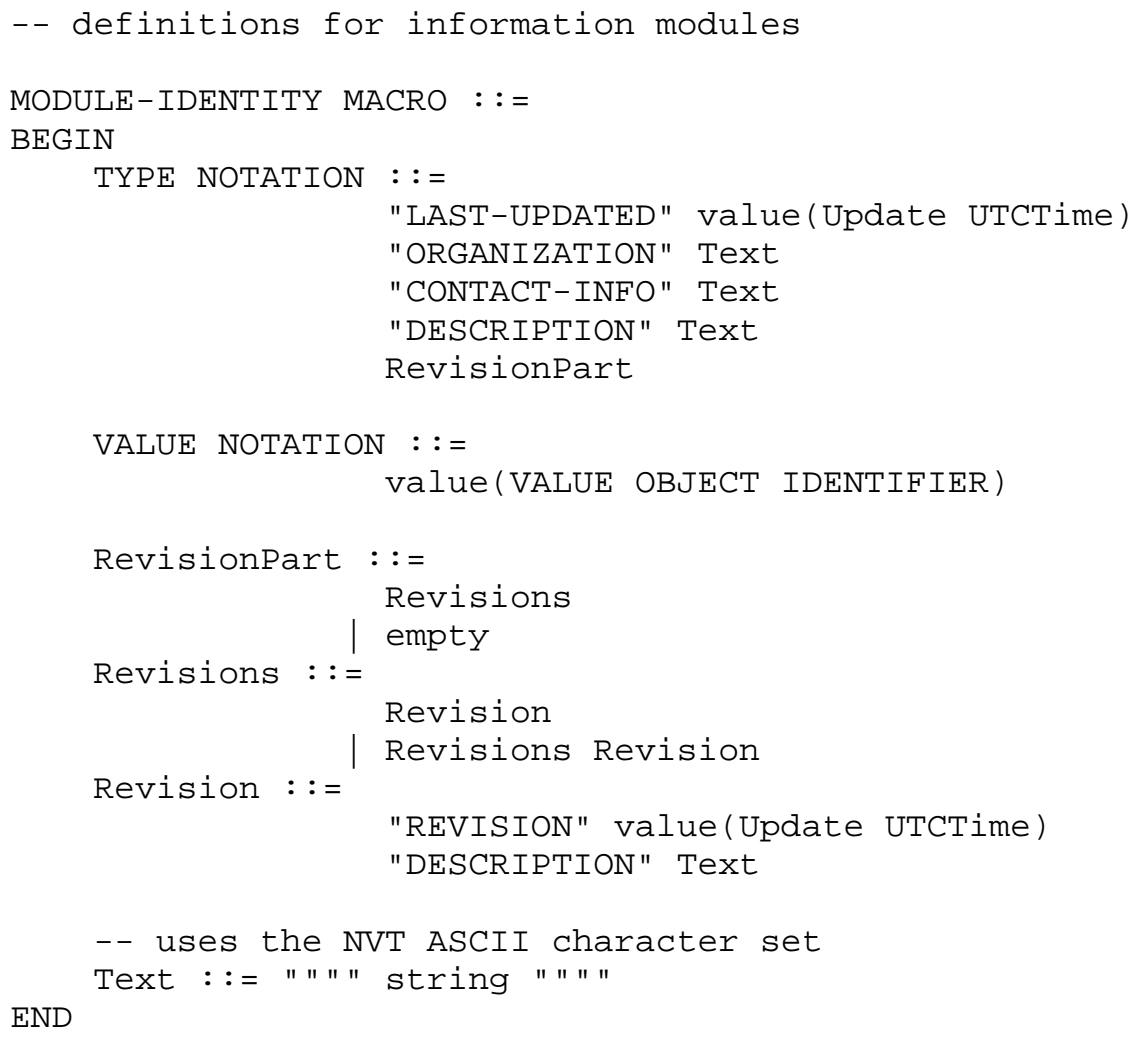




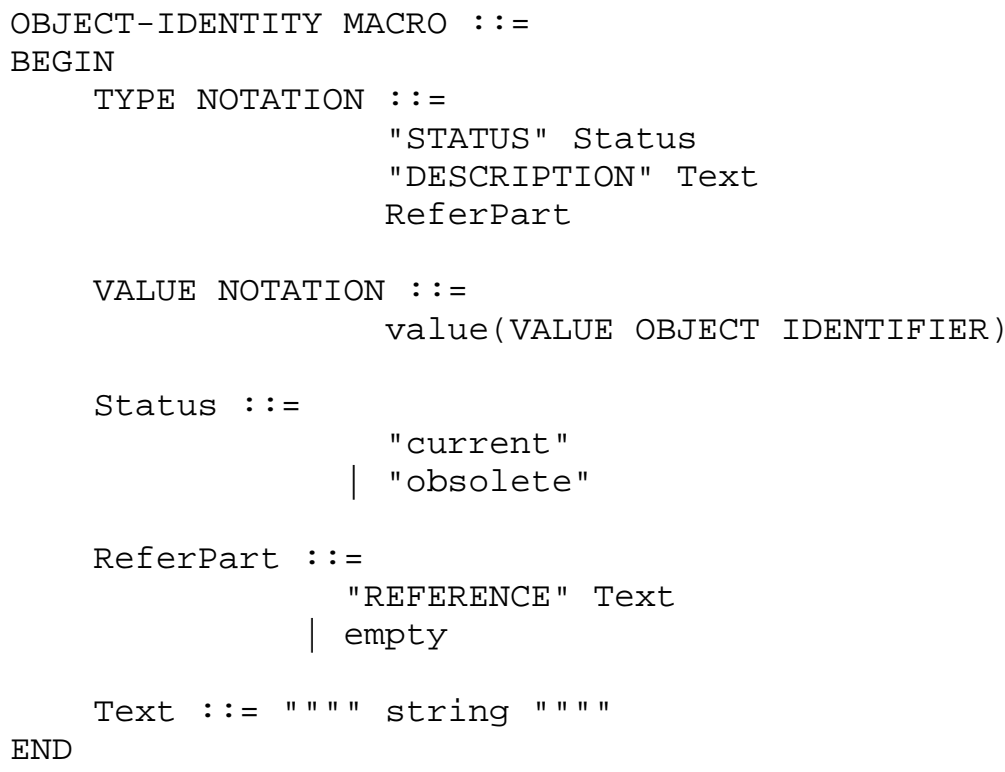




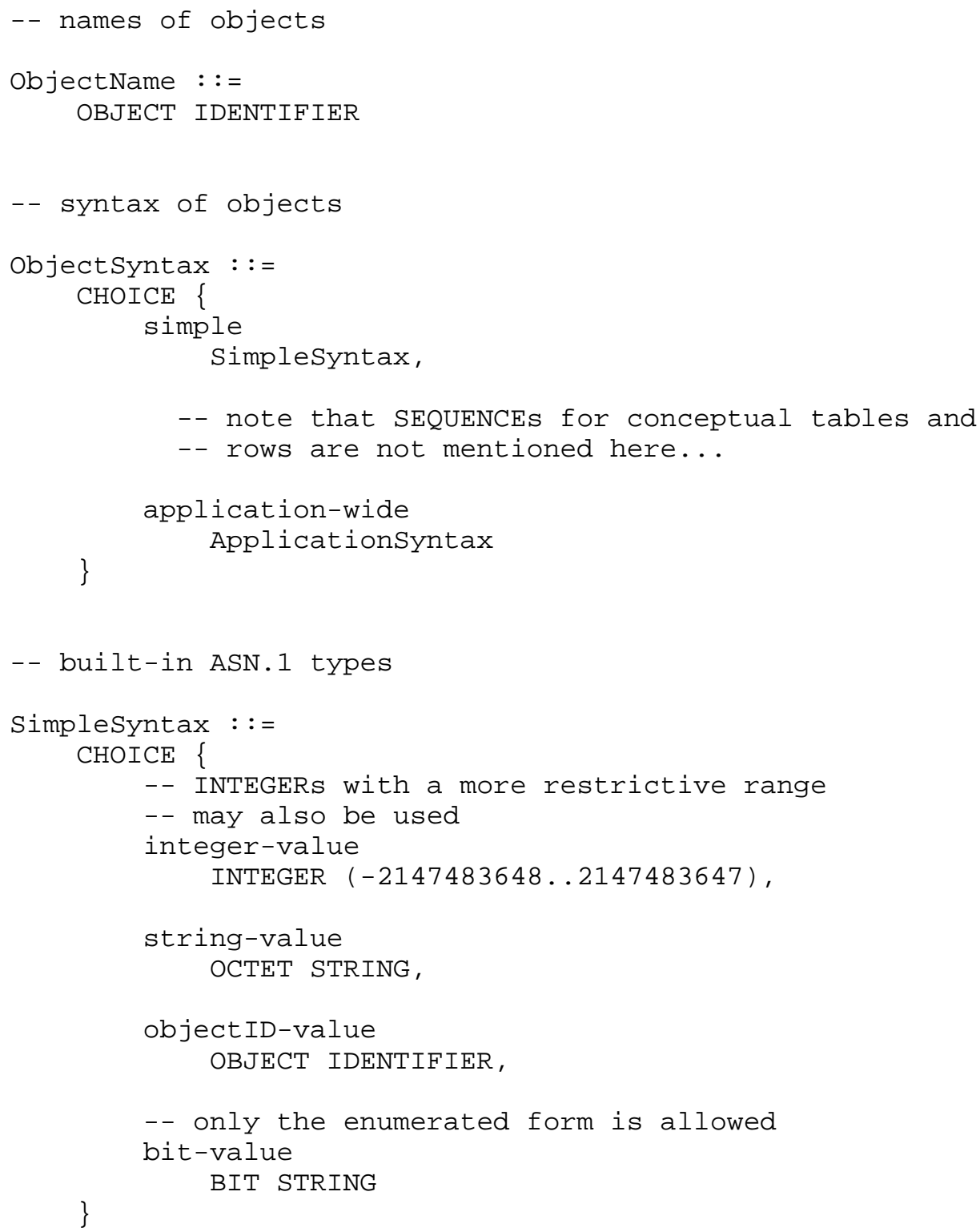


-- indistinguishable from INTEGER, but never needs more than -- 32-bits for a two's complement representation Integer32 : :=

[UNIVERSAL 2]

IMPLICIT INTEGER $(-2147483648 \ldots 2147483647)$

-- application-wide types

ApplicationSyntax : := CHOICE \{

ipAddress-value

IpAddress,

counter-value

Counter32,

gauge-value

Gauge 32,

timeticks-value

TimeTicks,

arbitrary-value

Opaque,

nsapAddress-value

NsapAddress,

big-counter-value

Counter64,

unsigned-integer-value

UInteger 32

\}

-- in network-byte order

-- (this is a tagged type for historical reasons)

IpAddress : :=

[APPLICATION 0]

IMPLICIT OCTET STRING (SIZE (4)) 


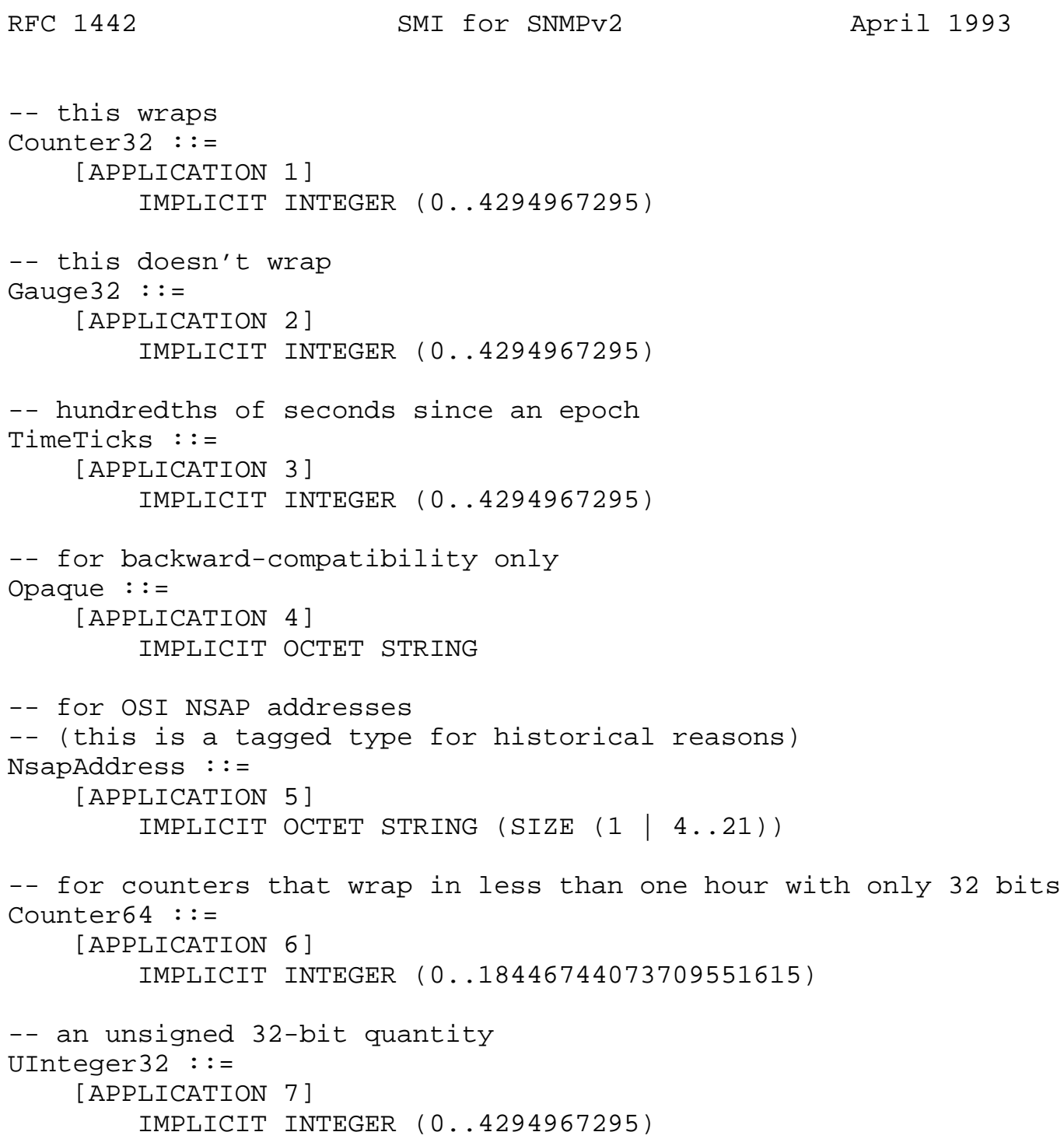




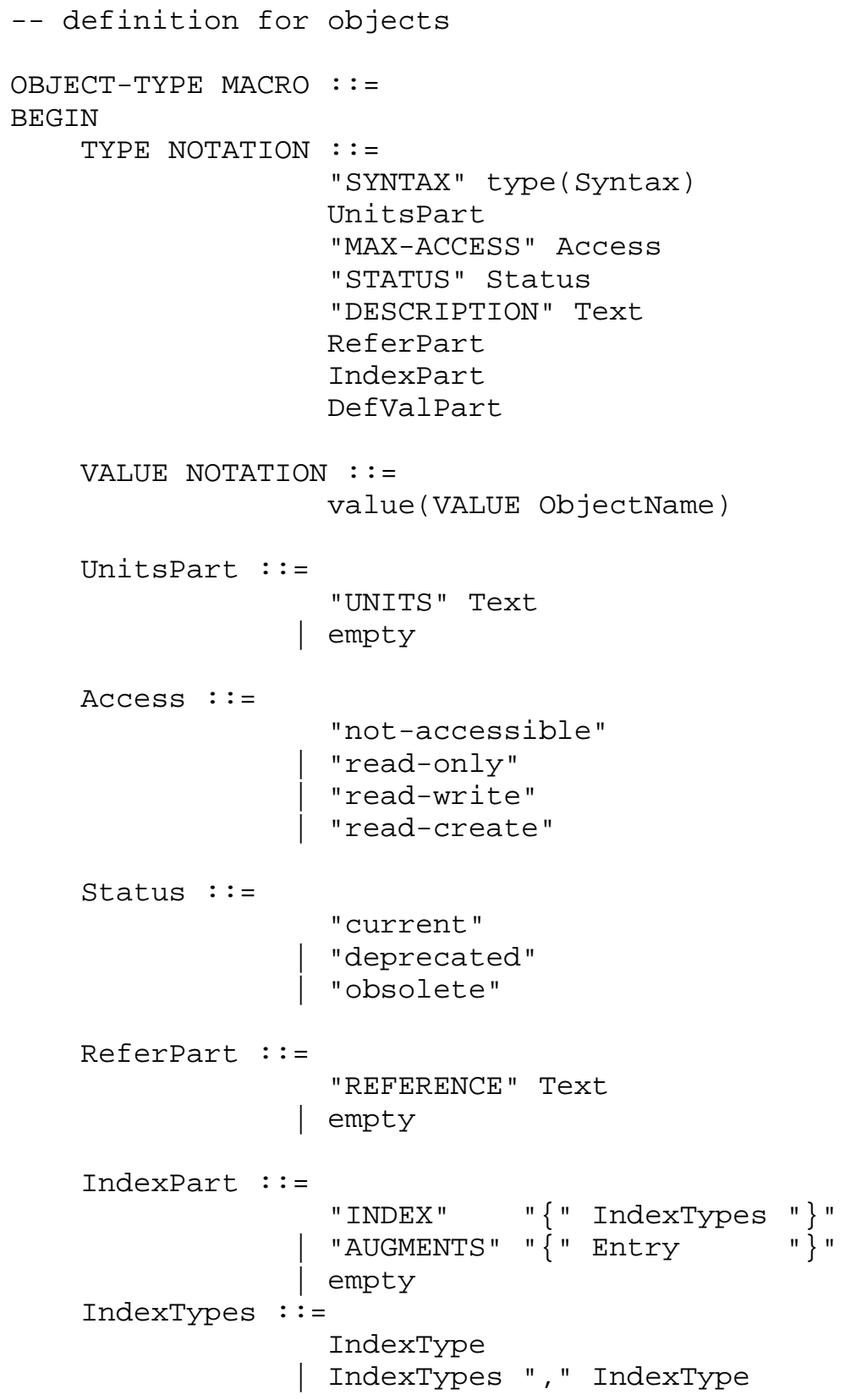




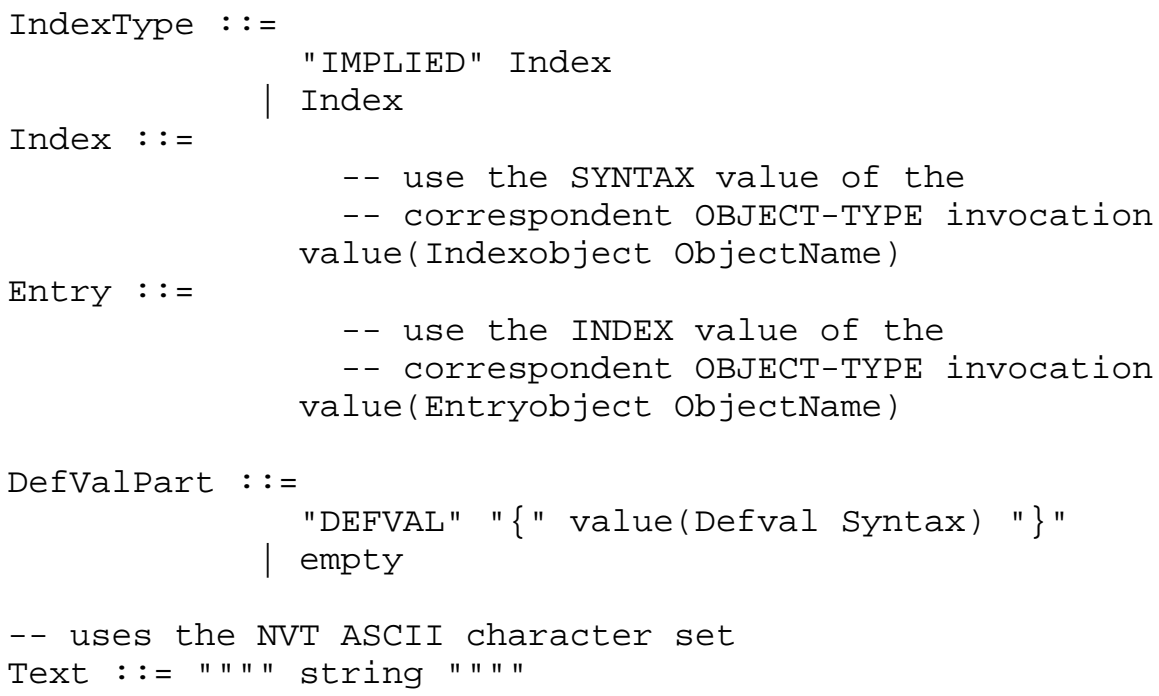




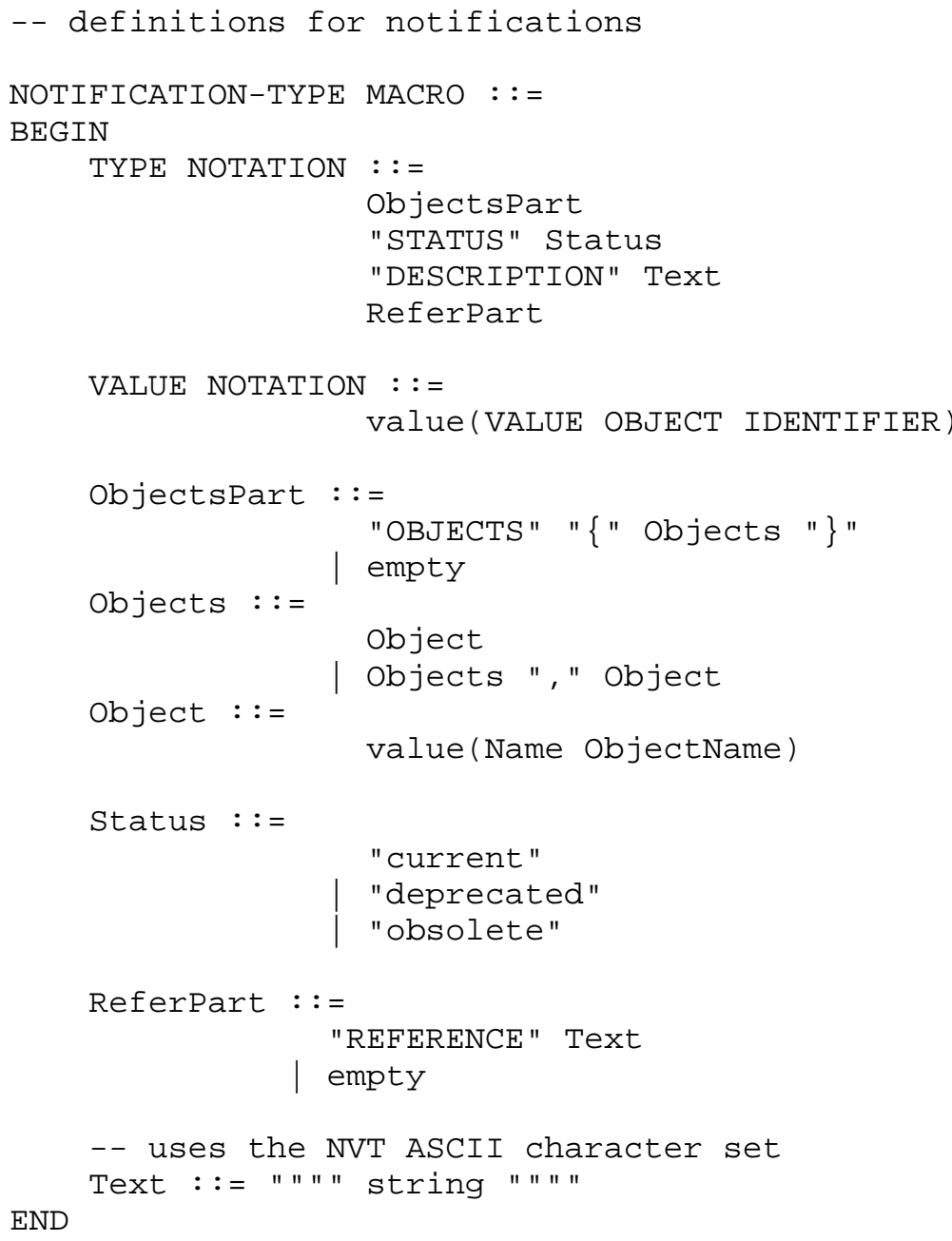

END 


\section{Information Modules}

An "information module" is an ASN.1 module defining information relating to network management.

The SMI describes how to use a subset of ASN.1 to define an information module. Further, additional restrictions are placed on "standard" information modules. It is strongly recommended that "enterprise-specific" information modules also adhere to these restrictions.

Typically, there are three kinds of information modules:

(1) MIB modules, which contain definitions of inter-related managed objects, make use of the OBJECT-TYPE and NOTIFICATION-TYPE macros;

(2) compliance statements for MIB modules, which make use of the MODULE-COMPLIANCE and OBJECT-GROUP macros [2]; and,

(3) capability statements for agent implementations which make use of the AGENT-CAPABILITIES macros [2].

This classification scheme does not imply a rigid taxonomy . For example, a "standard" information module might include definitions of managed objects and a compliance statement. Similarly, an "enterprise-specific" information module might include definitions of managed objects and a capability statement. Of course, a "standard" information module may not contain capability statements.

All information modules start with exactly one invocation of the MODULE-IDENTITY macro, which provides contact and revision history. This invocation must appear immediately after any IMPORTs or EXPORTs statements.

\subsection{Macro Invocation}

Within an information module, each macro invocation appears as:

$$
\text { <descriptor > <macro> <clauses }>: \text { : }<\text { value }>
$$

where <descriptor> corresponds to an ASN.1 identifier, <macro> 
names the macro being invoked, and <clauses> and <value> depend on the definition of the macro.

An ASN.1 identifier consists of one or more letters, digits, or hyphens. The initial character must be a lower-case letter, and the final character may not be a hyphen. Further, a hyphen may not be immediatedly followed by another hyphen.

For all descriptors appearing in an information module, the descriptor shall be unique and mnemonic, and shall not exceed 64 characters in length. This promotes a common language for humans to use when discussing the information module and also facilitates simple table mappings for user-interfaces.

The set of descriptors defined in all "standard" information modules shall be unique. Further, within any information module, the hyphen is not allowed as a character in any descriptor.

Finally, by convention, if the descriptor refers to an object with a SYNTAX clause value of either Counter32 or Counter64, then the descriptor used for the object should denote plurality.

\subsubsection{Textual Clauses}

Some clauses in a macro invocation may take a textual value (e.g., the DESCRIPTION clause). Note that, in order to conform to the ASN.1 syntax, the entire value of these clauses must be enclosed in double quotation marks, and therefore cannot itself contain double quotation marks, although the value may be multi-line.

\subsection{IMPORTing Symbols}

To reference an external object, the IMPORTS statement must be used to identify both the descriptor and the module defining the descriptor.

Note that when symbols from "enterprise-specific" information modules are referenced (e.g., a descriptor), there is the possibility of collision. As such, if different objects with the same descriptor are IMPORTed, then this ambiguity is 
resolved by prefixing the descriptor with the name of the information module and a dot ("."), i.e.,

"module.descriptor"

(All descriptors must be unique within any information module.)

Of course, this notation can be used even when there is no collision when IMPORTing symbols.

Finally, the IMPORTS statement may not be used to import an ASN.1 named type which corresponds to either the SEQUENCE or SEQUENCE OF type. 


\section{Naming Hierarchy}

The root of the subtree administered by the Internet Assigned Numbers Authority (IANA) for the Internet is:

$$
\text { internet OBJECT IDENTIFIER }::=\left\{\begin{array}{lllll}
\text { iso } & 3 & 6 & 1
\end{array}\right\}
$$

That is, the Internet subtree of OBJECT IDENTIFIERs starts with the prefix:

$$
1.3 \cdot 6 \cdot 1
$$

Several branches underneath this subtree are used for network management :

$$
\begin{array}{lll}
\text { mgmt } & \text { OBJECT IDENTIFIER }::=\{\text { internet } 2\} \\
\text { experimental } & \text { OBJECT IDENTIFIER }::=\{\text { internet } 3\} \\
\text { private } & \text { OBJECT IDENTIFIER }::=\{\text { internet } 4\} \\
\text { enterprises } & \text { OBJECT IDENTIFIER }::=\{\text { private } 1\}
\end{array}
$$

However, the SMI does not prohibit the definition of objects in other portions of the object tree.

The mgmt (2) subtree is used to identify "standard" objects.

The experimental(3) subtree is used to identify objects being designed by working groups of the IETF. If an information module produced by a working group becomes a "standard" information module, then at the very beginning of its entry onto the Internet standards track, the objects are moved under the mgmt (2) subtree.

The private(4) subtree is used to identify objects defined unilaterally. The enterprises(1) subtree beneath private is used, among other things, to permit providers of networking subsystems to register models of their products. 


\section{Mapping of the MODULE-IDENTITY macro}

The MODULE-IDENTITY macro is used to provide contact and revision history for each information module. It must appear exactly once in every information module. It should be noted that the expansion of the MODULE-IDENTITY macro is something which conceptually happens during implementation and not during run-time.

5.1. Mapping of the LAST-UPDATED clause

The LAST-UPDATED clause, which must be present, contains the date and time that this information module was last edited.

\subsection{Mapping of the ORGANIZATION clause}

The ORGANIZATION clause, which must be present, contains a textual description of the organization under whose auspices this information module was developed.

\subsection{Mapping of the CONTACT-INFO clause}

The CONTACT-INFO clause, which must be present, contains the name, postal address, telephone number, and electronic mail address of the person to whom technical queries concerning this information module should be sent.

5.4. Mapping of the DESCRIPTION clause

The DESCRIPTION clause, which must be present, contains a high-level textual description of the contents of this information module.

\subsection{Mapping of the REVISION clause}

The REVISION clause, which need not be present, is repeatedly used to describe the revisions made to this information

module, in reverse chronological order. Each instance of this clause contains the date and time of the revision. 
5.6. Mapping of the DESCRIPTION clause

The DESCRIPTION clause, which must be present for each

REVISION clause, contains a high-level textual description of the revision identified in that REVISION clause.

5.7. Mapping of the MODULE-IDENTItY value

The value of an invocation of the MODULE-IDENTITY macro is an OBJECT IDENTIFIER. As such, this value may be authoritatively used when referring to the information module containing the invocation. 


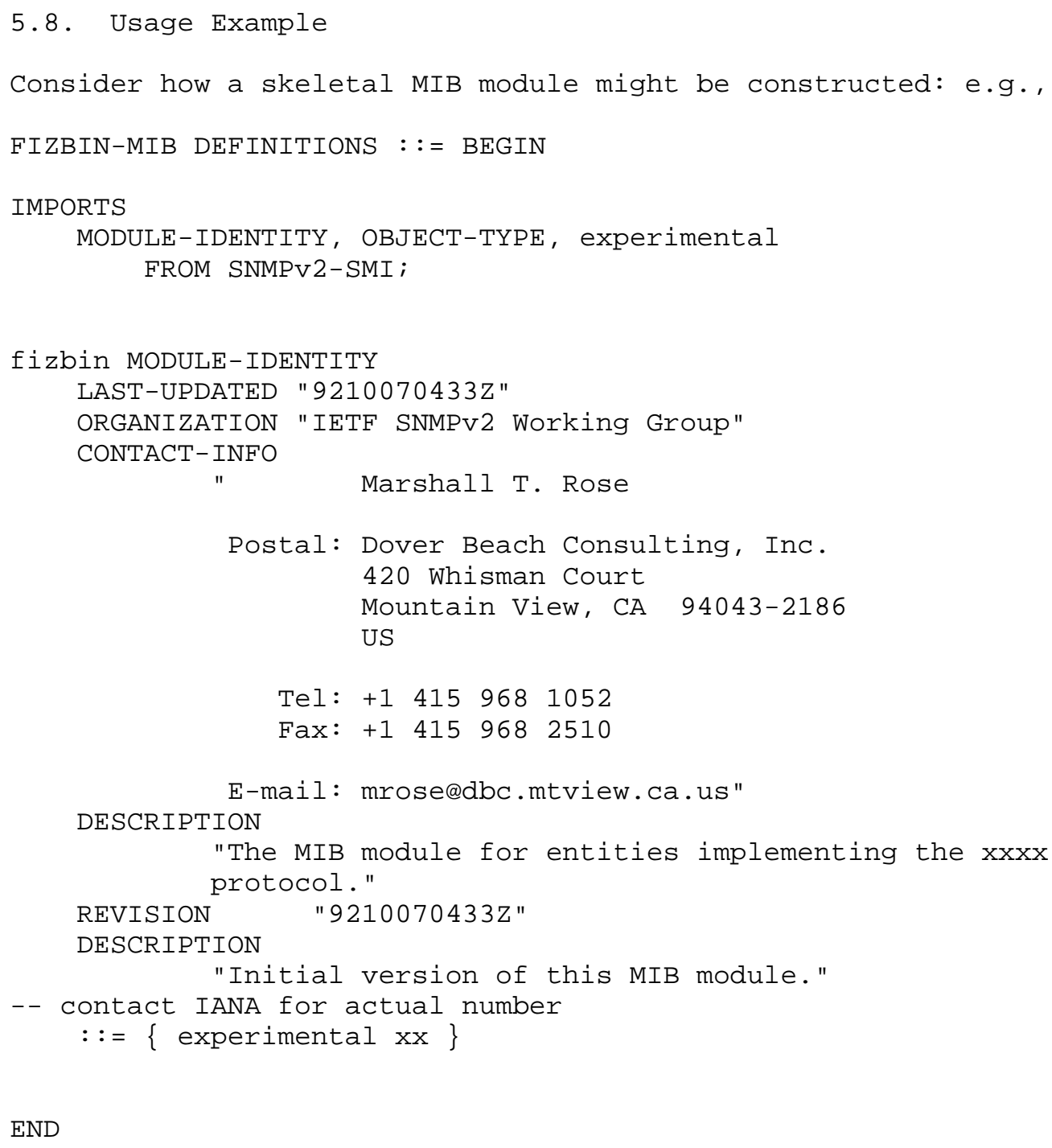

END 


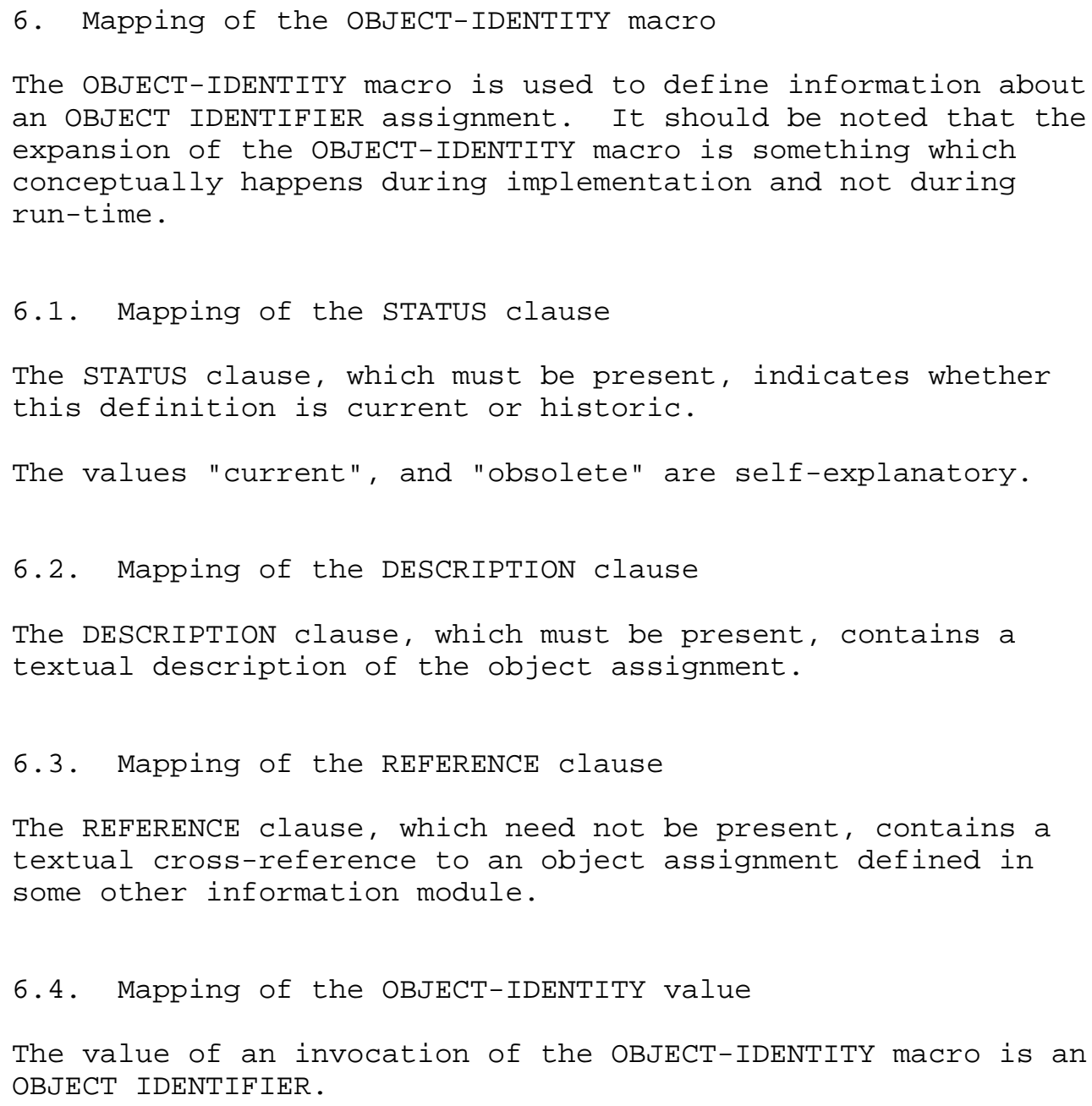


6.5. Usage Example

Consider how an OBJECT IDENTIFIER assignment might be made: e.g.,

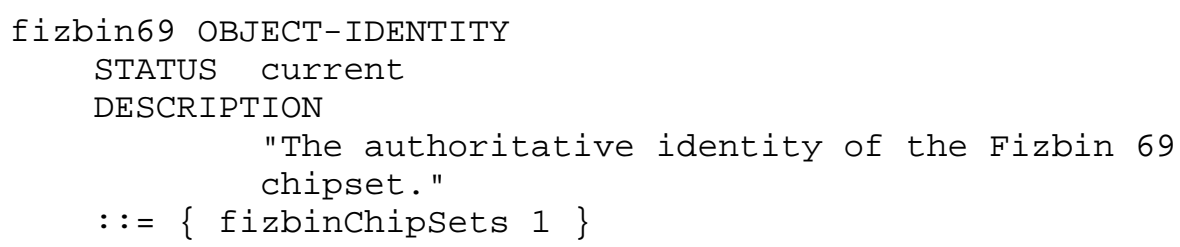




\section{Mapping of the OBJECT-TYPE macro}

The OBJECT-TYPE macro is used to define a managed object. It should be noted that the expansion of the OBJECT-TYPE macro is something which conceptually happens during implementation and not during run-time.

\subsection{Mapping of the SYNTAX clause}

The SYNTAX clause, which must be present, defines the abstract data structure corresponding to that object. The data structure must be one of the alternatives defined in the ObjectSyntax CHOICE.

Full ASN.1 sub-typing is allowed, as appropriate to the underingly ASN.1 type, primarily as an aid to implementors in understanding the meaning of the object. Any such restriction on size, range, enumerations or repertoire specified in this clause represents the maximal level of support which makes "protocol sense". Of course, sub-typing is not allowed for the Counter 32 or Counter64 types, but is allowed for the Gauge 32 type.

The semantics of ObjectSyntax are now described.

\subsubsection{Integer32 and INTEGER}

The Integer32 type represents integer-valued information between $-2^{\wedge} 31$ and $2^{\wedge} 31-1$ inclusive $(-2147483648$ to 2147483647 decimal). This type is indistinguishable from the INTEGER type.

The INTEGER type may also be used to represent integer-valued information, if it contains named-number enumerations, or if it is sub-typed to be more constrained than the Integer32 type. In the former case, only those named-numbers so enumerated may be present as a value. Note that although it is recommended that enumerated values start at 1 and be numbered contiguously, any valid value for Integer32 is allowed for an enumerated value and, further, enumerated values needn't be contiguously assigned. 
Finally, the hyphen character is not allowed as a part of the label name for any named-number enumeration.

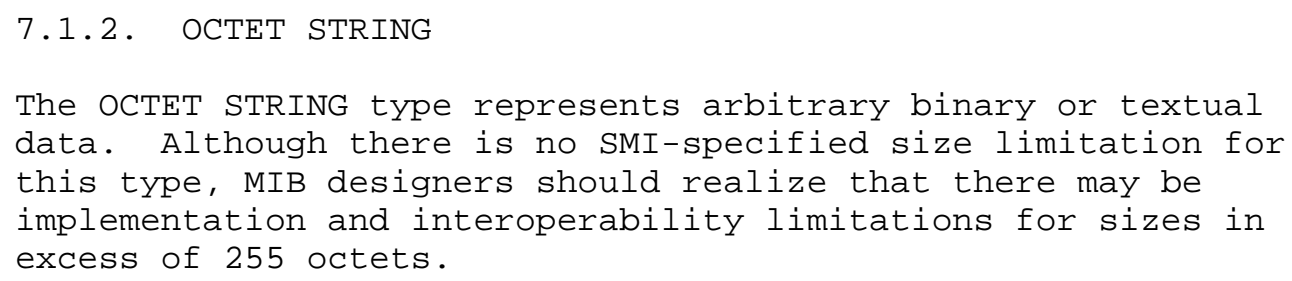

\subsubsection{OBJECT IDENTIFIER}

The OBJECT IDENTIFIER type represents administratively assigned names. Any instance of this type may have at most 128 sub-identifiers. Further, each sub-identifier must not exceed the value 2^32-1 (4294967295 decimal).

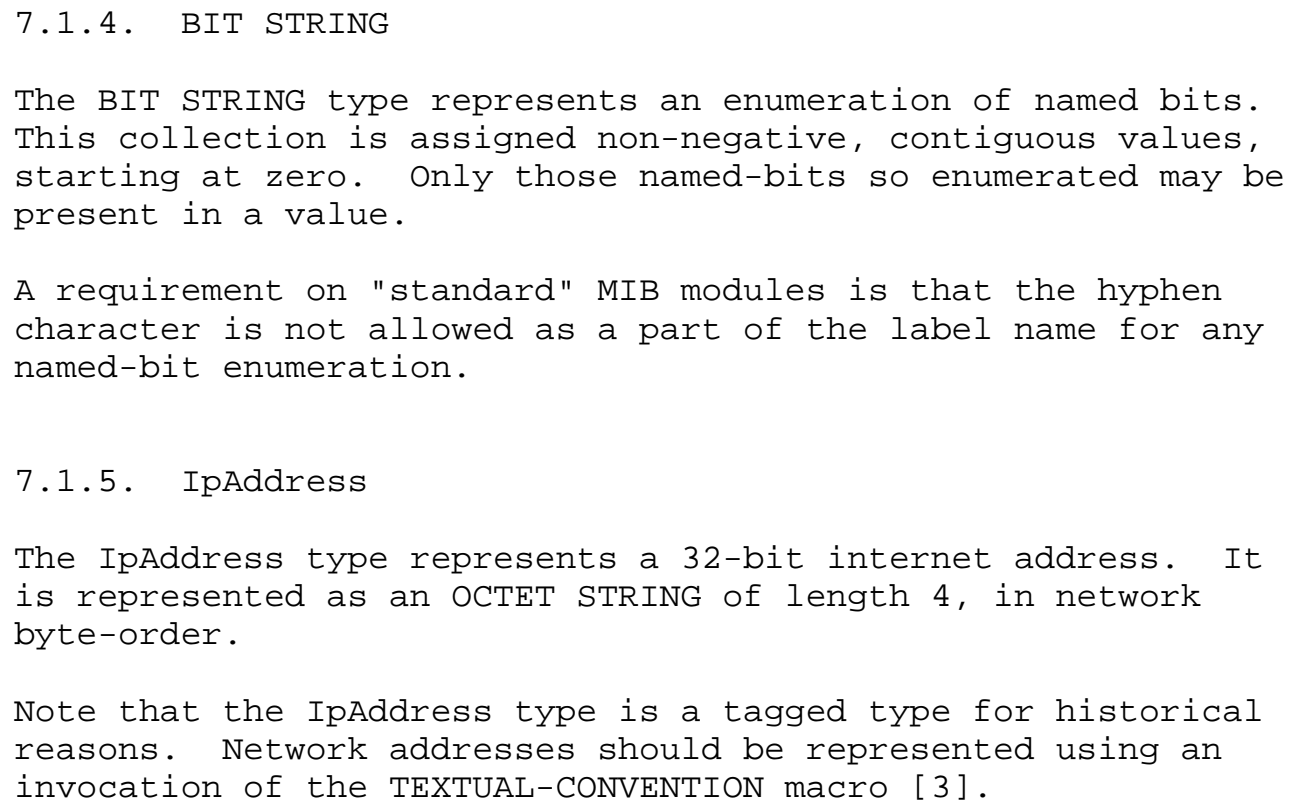




\subsubsection{Counter32}

The Counter32 type represents a non-negative integer which monotonically increases until it reaches a maximum value of 2^32-1 (4294967295 decimal), when it wraps around and starts increasing again from zero.

Counters have no defined "initial" value, and thus, a single value of a Counter has (in general) no information content. Discontinuities in the monotonically increasing value normally occur at re-initialization of the management system, and at other times as specified in the description of an object-type using this ASN.1 type. If such other times can occur, for example, the creation of an object instance at times other than re-initialization, then a corresponding object should be defined with a SYNTAX clause value of Timestamp (a textual convention defined in [3]) indicating the time of the last discontinuity.

The value of the MAX-ACCESS clause for objects with a SYNTAX clause value of Counter 32 is always "read-only".

A DEFVAL clause is not allowed for objects with a SYNTAX clause value of Counter32.

\subsubsection{Gauge32}

The Gauge32 type represents a non-negative integer, which may increase or decrease, but shall never exceed a maximum value. The maximum value can not be greater than 2^32-1 (4294967295 decimal). The value of a Gauge has its maximum value whenever the information being modeled is greater or equal to that maximum value; if the information being modeled subsequently decreases below the maximum value, the Gauge also decreases.

\subsubsection{TimeTicks}

The TimeTicks type represents a non-negative integer which represents the time, modulo 2^32 (4294967296 decimal), in hundredths of a second between two epochs. When objects are defined which use this ASN.1 type, the description of the object identifies both of the reference epochs. 
For example, [3] defines the Timestamp textual convention which is based on the TimeTicks type. With a Timestamp, the first reference epoch is defined as when MIB-II's sysUpTime [7] was zero, and the second reference epoch is defined as the current value of sysUpTime.

\subsubsection{Opaque}

The Opaque type is provided solely for backward-compatibility, and shall not be used for newly-defined object types.

The Opaque type supports the capability to pass arbitrary ASN.1 syntax. A value is encoded using the ASN.1 Basic Encoding Rules [4] into a string of octets. This, in turn, is encoded as an OCTET STRING, in effect "double-wrapping" the original ASN.1 value.

Note that a conforming implementation need only be able to accept and recognize opaquely-encoded data. It need not be able to unwrap the data and then interpret its contents.

A requirement on "standard" MIB modules is that no object may have a SYNTAX clause value of Opaque.

\subsubsection{NsapAddress}

The NsapAddress type represents an OSI address as a variablelength OCTET STRING. The first octet of the string contains a binary value in the range of $0 . .20$, and indicates the length in octets of the NSAP. Following the first octet, is the NSAP, expressed in concrete binary notation, starting with the most significant octet. A zero-length NSAP is used as a "special" address meaning "the default NSAP" (analogous to the IP address of 0.0.0.0). Such an NSAP is encoded as a single octet, containing the value 0 . All other NSAPs are encoded in at least 4 octets.

Note that the NsapAddress type is a tagged type for historical reasons. Network addresses should be represented using an invocation of the TEXTUAL-CONVENTION macro [3] . 


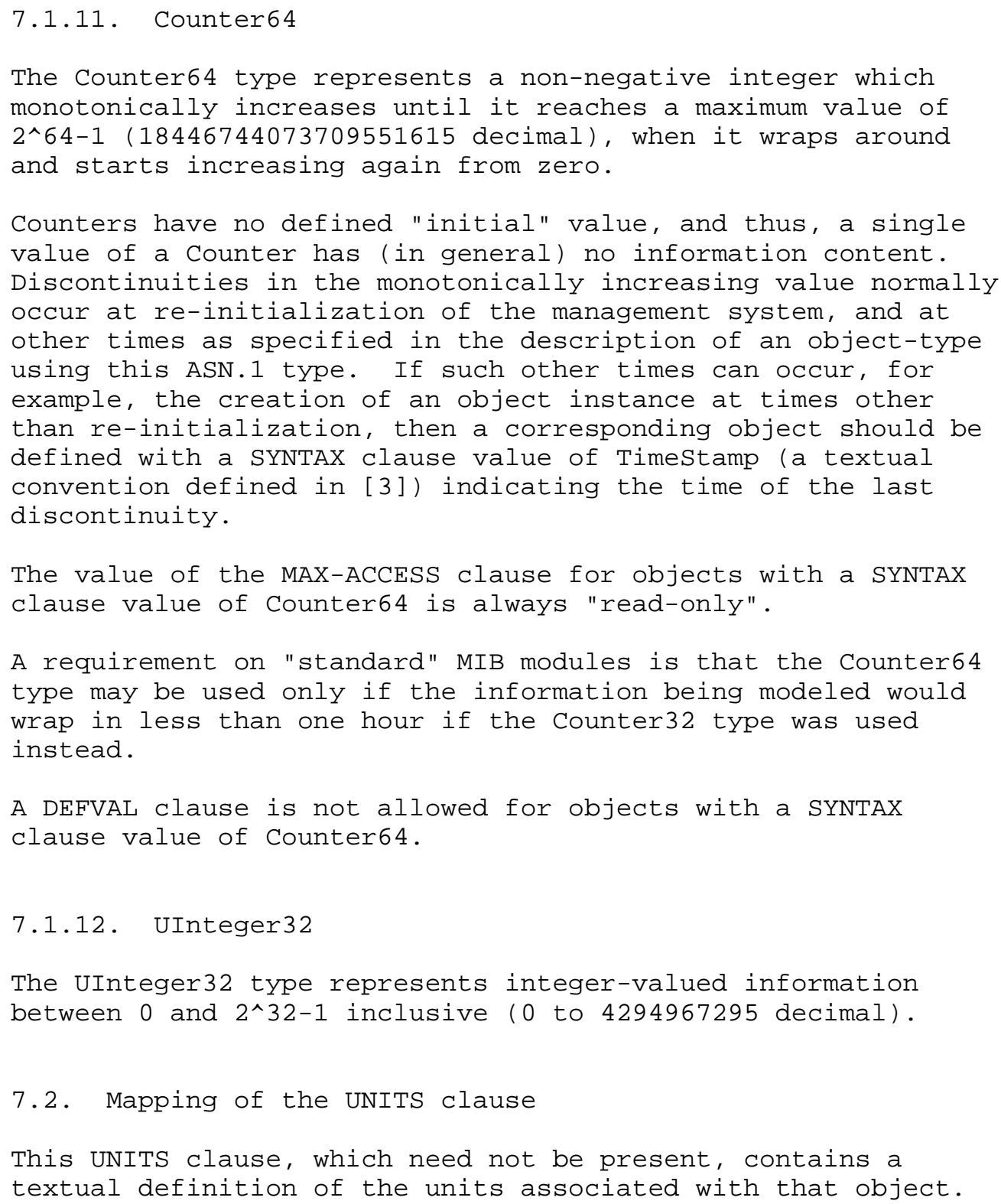




\subsection{Mapping of the MAX-ACCESS clause}

The MAX-ACCESS clause, which must be present, defines whether it makes "protocol sense" to read, write and/or create an instance of the object. This is the maximal level of access for the object. (This maximal level of access is independent of any administrative authorization policy.)

The value "read-write" indicates that read and write access make "protocol sense", but create does not. The value "readcreate" indicates that read, write and create access make "protocol sense". The value "not-accessible" indicates either an auxiliary object (see section 7.7) or an object which is accessible only via a notificationn (e.g., snmptrapold [5]).

These values are ordered, from least to greatest: "notaccessible", "read-only", "read-write", "read-create".

If any columnar object in a conceptual row has "read-create" as its maximal level of access, then no other columnar object of the same conceptual row may have a maximal access of "read-write". (Note that "read-create" is a superset of "read-write". )

\subsection{Mapping of the STATUS clause}

The STATUS clause, which must be present, indicates whether this definition is current or historic.

The values "current", and "obsolete" are self-explanatory. The "deprecated" value indicates that the object is obsolete, but that an implementor may wish to support that object to foster interoperability with older implementations.

\subsection{Mapping of the DESCRIPTION clause}

The DESCRIPTION clause, which must be present, contains a textual definition of that object which provides all semantic definitions necessary for implementation, and should embody any information which would otherwise be communicated in any ASN.1 commentary annotations associated with the object. 
7.6. Mapping of the REFERENCE clause

The REFERENCE clause, which need not be present, contains a textual cross-reference to an object defined in some other information module. This is useful when de-osifying a MIB module produced by some other organization.

\subsection{Mapping of the INDEX clause}

The INDEX clause, which must be present if that object corresponds to a conceptual row (unless an AUGMENTS clause is present instead), and must be absent otherwise, defines instance identification information for the columnar objects subordinate to that object.

Management operations apply exclusively to scalar objects. However, it is convenient for developers of management applications to impose imaginary, tabular structures on the ordered collection of objects that constitute the MIB. Each such conceptual table contains zero or more rows, and each row may contain one or more scalar objects, termed columnar objects. This conceptualization is formalized by using the OBJECT-TYPE macro to define both an object which corresponds to a table and an object which corresponds to a row in that table. A conceptual table has SYNTAX of the form:

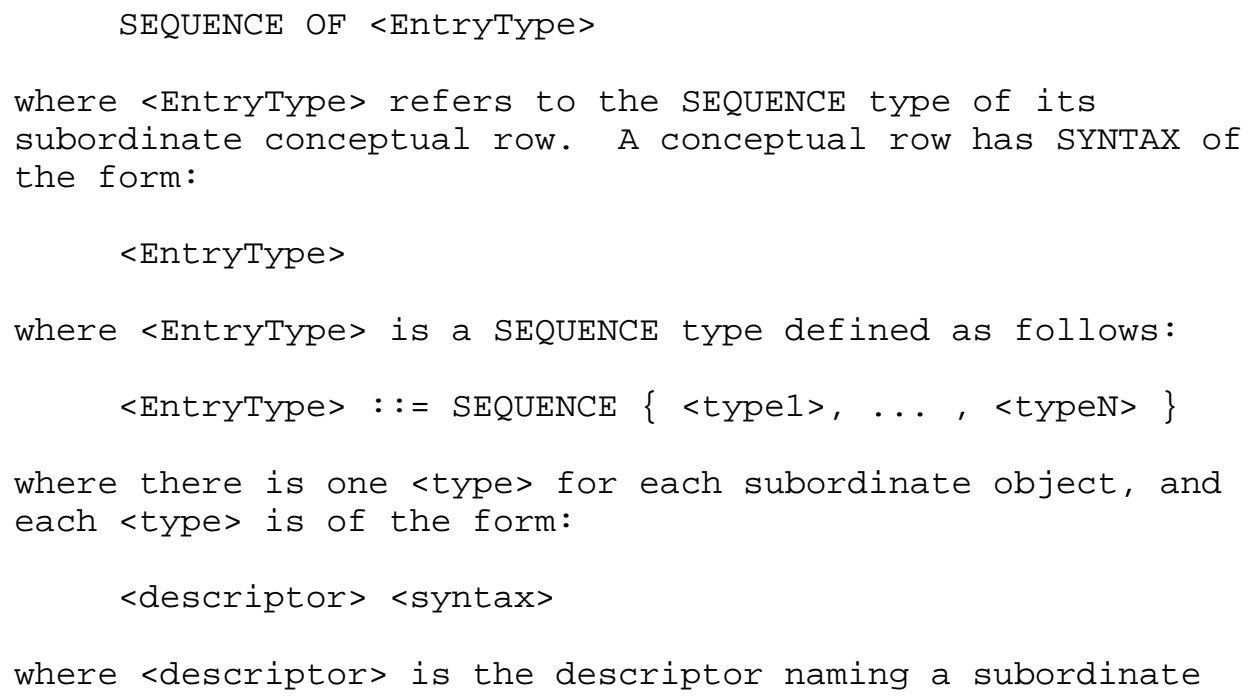


object, and <syntax> has the value of that subordinate object's SYNTAX clause, optionally omitting the sub-typing information. Further, these ASN.1 types are always present (the DEFAULT and OPTIONAL clauses are disallowed in the SEQUENCE definition). The MAX-ACCESS clause for conceptual tables and rows is "not-accessible".

For leaf objects which are not columnar objects, instances of the object are identified by appending a sub-identifier of zero to the name of that object. Otherwise, the INDEX clause of the conceptual row object superior to a columnar object defines instance identification information.

The instance identification information in an INDEX clause must specify object(s) such that value(s) of those object(s) will unambiguously distinguish a conceptual row. The syntax of those objects indicate how to form the instance-identifier:

(1) integer-valued: a single sub-identifier taking the integer value (this works only for non-negative integers);

(2) string-valued, fixed-length strings (or variable-length preceded by the IMPLIED keyword): ' $n$ ' sub-identifiers, where ' $n$ ' is the length of the string (each octet of the string is encoded in a separate sub-identifier);

(3) string-valued, variable-length strings (not preceded by the IMPLIED keyword): ' $n+1$ ' sub-identifiers, where ' $\mathrm{n}$ ' is the length of the string (the first sub-identifier is ' $n$ ' itself, following this, each octet of the string is encoded in a separate sub-identifier);

(4) object identifier-valued: ' $n+1$ ' sub-identifiers, where ' $n$ ' is the number of sub-identifiers in the value (the first sub-identifier is ' $n$ ' itself, following this, each sub-identifier in the value is copied);

(5) IpAddress-valued: 4 sub-identifiers, in the familiar a.b.c.d notation.

(6) NsapAddress-valued: ' $n$ ' sub-identifiers, where ' $\mathrm{n}$ ' is the length of the value (each octet of the value is encoded in a separate sub-identifier); 
Note that the IMPLIED keyword can only be present for objects having a variable-length syntax (e.g., variable-length strings or object identifier-valued objects). Further, the IMPLIED keyword may appear at most once within the INDEX clause, and if so, is associated with the right-most object having a variable-length syntax. Finally, the IMPLIED keyword may not be used on a variable-length string object if that string might have a value of zero-length.

Instances identified by use of integer-valued objects should be numbered starting from one (i.e., not from zero). The use of zero as a value for an integer-valued index object should be avoided, except in special cases.

Objects which are both specified in the INDEX clause of a conceptual row and also columnar objects of the same conceptual row are termed auxiliary objects. The MAX-ACCESS clause for newly-defined auxiliary objects is "notaccessible". However, a conceptual row must contain at least one columnar object which is not an auxiliary object (i.e., the value of the MAX-ACCESS clause for such an object is either "read-only" or "read-create").

Note that objects specified in a conceptual row's INDEX clause need not be columnar objects of that conceptual row. In this situation, the DESCRIPTION clause of the conceptual row must include a textual explanation of how the objects which are included in the INDEX clause but not columnar objects of that conceptual row, are used in uniquely identifying instances of the conceptual row's columnar objects.

\subsubsection{Creation and Deletion of Conceptual Rows}

For newly-defined conceptual rows which allow the creation of new object instances and the deletion of existing object instances, there should be one columnar object with a SYNTAX clause value of Rowstatus (a textual convention defined in [3]) and a MAX-ACCESS clause value of read-create. By convention, this is termed the status column for the conceptual row. 


\subsection{Mapping of the AUGMENTS clause}

The AUGMENTS clause, which must not be present unless the object corresponds to a conceptual row, is an alternative to the INDEX clause. Every object corresponding to a conceptual row has either an INDEX clause or an AUGMENTS clause.

If an object corresponding to a conceptual row has an INDEX clause, that row is termed a base conceptual row; alternatively, if the object has an AUGMENTS clause, the row is said to be a conceptual row augmentation, where the AUGMENTS clause names the object corresponding to the base conceptual row which is augmented by this conceptual row extension. Instances of subordinate columnar objects of a conceptual row extension are identified according to the INDEX clause of the base conceptual row corresponding to the object named in the AUGMENTS clause. Further, instances of subordinate columnar objects of a conceptual row extension exist according to the same semantics as instances of subordinate columnar objects of the base conceptual row being augmented. As such, note that creation of a base conceptual row implies the correspondent creation of any conceptual row augmentations.

For example, a MIB designer might wish to define additional columns in an "enterprise-specific" MIB which logically extend a conceptual row in a "standard" MIB. The "standard" MIB definition of the conceptual row would include the INDEX clause and the "enterprise-specific" MIB would contain the definition of a conceptual row using the AUGMENTS clause.

Note that a base conceptual row may be augmented by multiple conceptual row extensions.

7.8.1. Relation between INDEX and AUGMENTS clauses

When defining instance identification information for a conceptual table:

(1) If there is a one-to-one correspondence between the conceptual rows of this table and an existing table, then the AUGMENTS clause should be used. 
(2) Otherwise, if there is a sparse relationship between the conceptuals rows of this table and an existing table, then an INDEX clause should be used which is identical to that in the existing table.

(3) Otherwise, auxiliary objects should be defined within the conceptual row for the new table, and those objects should be used within the INDEX clause for the conceptual row.

\subsection{Mapping of the DEFVAL clause}

The DEFVAL clause, which need not be present, defines an acceptable default value which may be used at the discretion of a SNMPV2 entity acting in an agent role when an object instance is created.

During conceptual row creation, if an instance of a columnar object is not present as one of the operands in the correspondent management protocol set operation, then the value of the DEFVAL clause, if present, indicates an acceptable default value that a SNMPv2 entity acting in an agent role might use.

The value of the DEFVAL clause must, of course, correspond to the SYNTAX clause for the object. If the value is an OBJECT IDENTIFIER, then it must be expressed as a single ASN.1 identifier, and not as a collection of sub-identifiers.

Note that if an operand to the management protocol set operation is an instance of a read-only object, then the error 'notWritable' [6] will be returned. As such, the DEFVAL clause can be used to provide an acceptable default value that a SNMPV2 entity acting in an agent role might use.

By way of example, consider the following possible DEFVAL clauses: 


\begin{tabular}{|c|c|}
\hline Objectsyntax & DEFVAL clause \\
\hline Integer 32 & 1 \\
\hline & -- same for Gauge32, TimeTicks, UInteger 32 \\
\hline INTEGER & valid -- enumerated value \\
\hline OCTET STRING & 'ffffffffffff'H \\
\hline OBJECT IDENTIFIER & sysDescr \\
\hline $\begin{array}{l}\text { BIT STRING } \\
\text { IpAddress }\end{array}$ & $\begin{array}{c}\{\text { primary, secondary }\}-- \text { enumerated values } \\
{\text { ' } \mathrm{C} 0210415^{\prime} \mathrm{H}--}^{192.33 .4 .21}\end{array}$ \\
\hline
\end{tabular}

Object types with SYNTAX of Counter32 and Counter64 may not have DEFVAL clauses, since they do not have defined initial values. However, it is recommended that they be initialized to zero.

\subsection{Mapping of the OBJECT-TYPE value}

The value of an invocation of the OBJECT-TYPE macro is the name of the object, which is an OBJECT IDENTIFIER, an administratively assigned name.

When an OBJECT IDENTIFIER is assigned to an object:

(1) If the object corresponds to a conceptual table, then only a single assignment, that for a conceptual row, is present immediately beneath that object. The administratively assigned name for the conceptual row object is derived by appending a sub-identifier of "1" to the administratively assigned name for the conceptual table.

(2) If the object corresponds to a conceptual row, then at least one assignment, one for each column in the conceptual row, is present beneath that object. The administratively assigned name for each column is derived by appending a unique, positive sub-identifier to the administratively assigned name for the conceptual row.

(3) Otherwise, no other OBJECT IDENTIFIERs which are subordinate to the object may be assigned.

Note that the final sub-identifier of any administratively assigned name for an object shall be positive. A zero-valued final sub-identifier is reserved for future use. 
Further note that although conceptual tables and rows are given administratively assigned names, these conceptual objects may not be manipulated in aggregate form by the management protocol. 


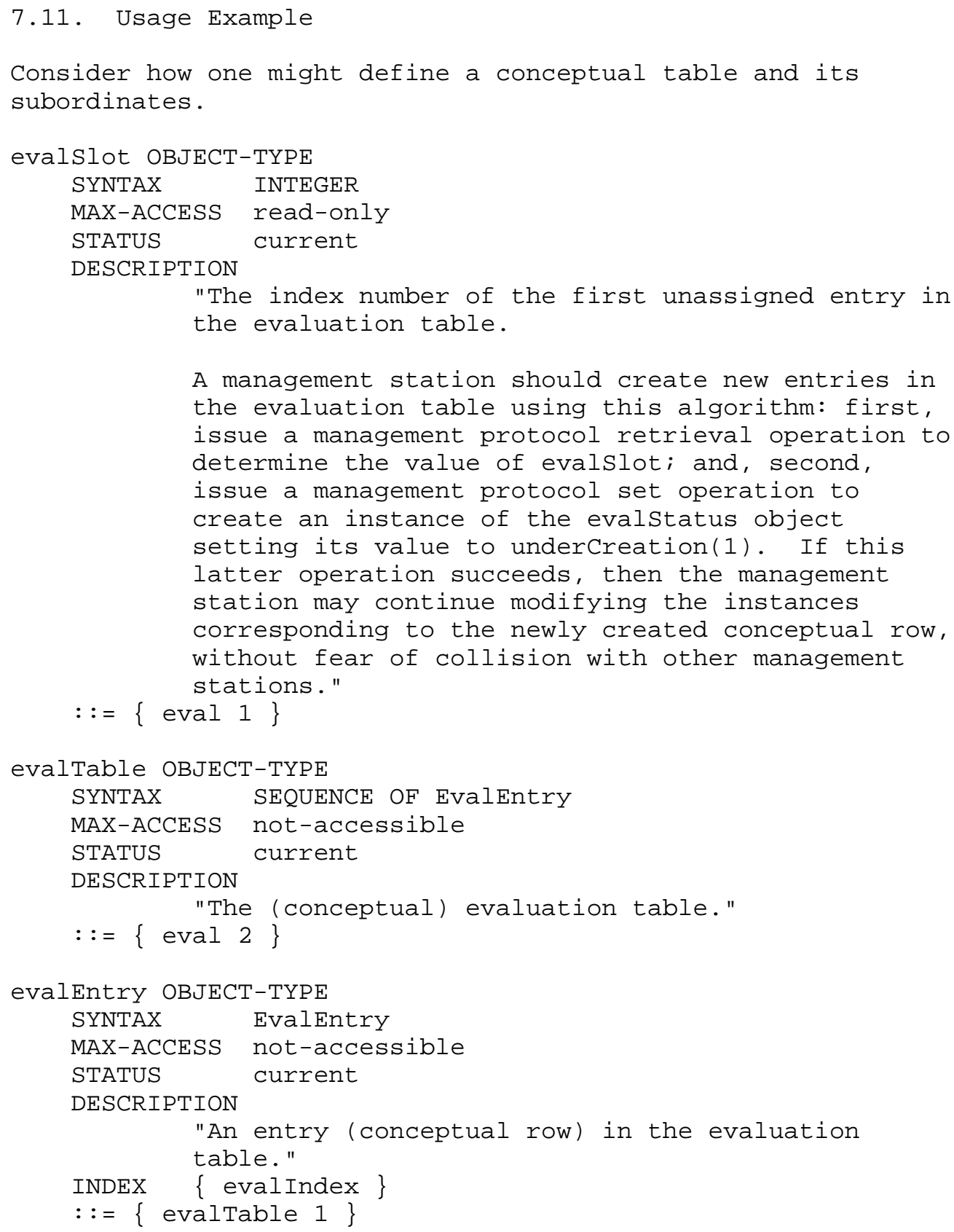




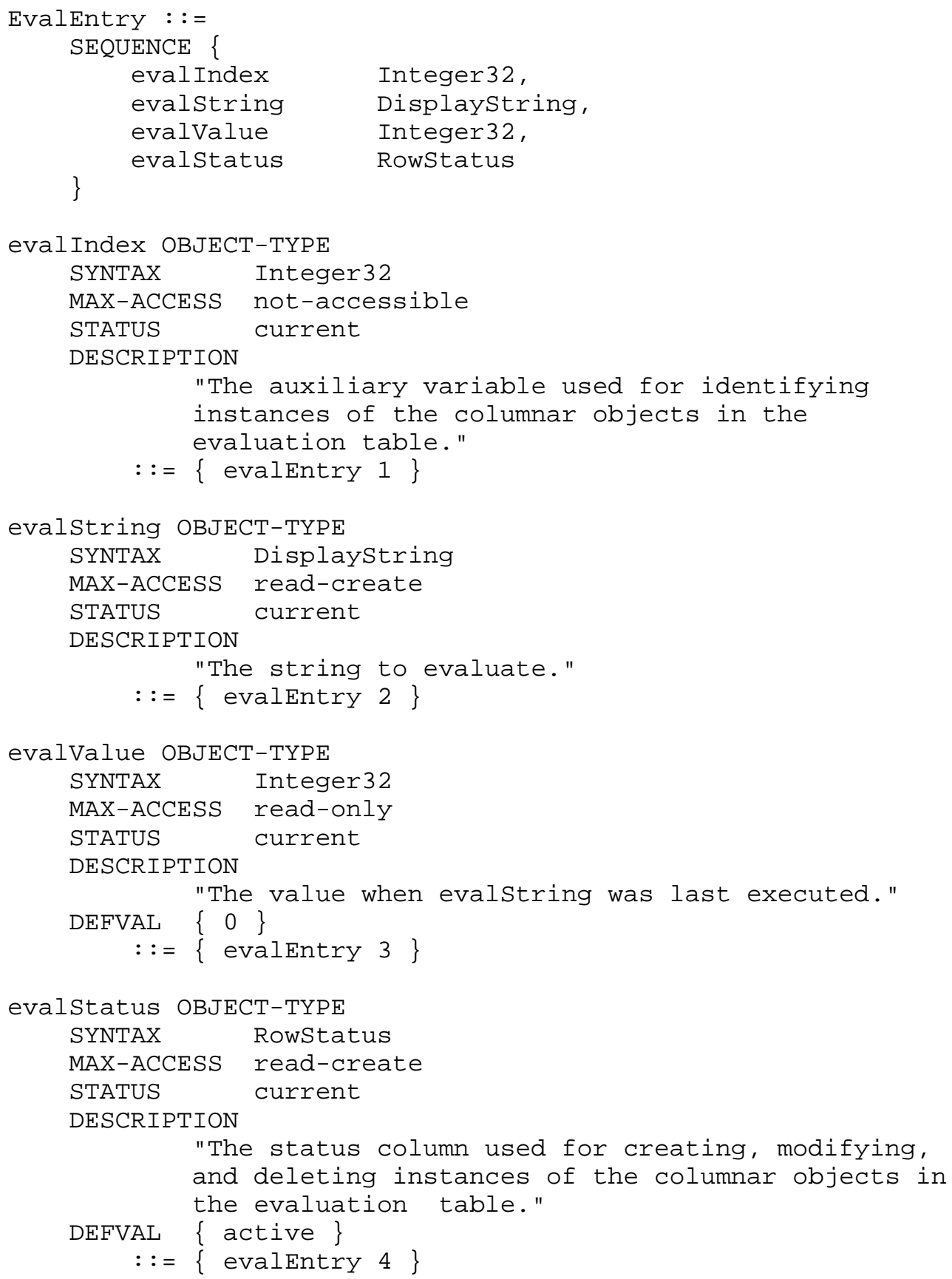




\section{Mapping of the NOTIFICATION-TYPE macro}

The NOTIFICATION-TYPE macro is used to define the information contained within an unsolicited transmission of management information (i.e., within either a SNMPV2-Trap-PDU or InformRequest-PDU). It should be noted that the expansion of the NOTIFICATION-TYPE macro is something which conceptually happens during implementation and not during run-time.

\subsection{Mapping of the OBJECTS clause}

The OBJECTS clause, which need not be present, defines the ordered sequence of MIB objects which are contained within every instance of the notification.

\subsection{Mapping of the STATUS clause}

The STATUS clause, which must be present, indicates whether this definition is current or historic.

The values "current", and "obsolete" are self-explanatory. The "deprecated" value indicates that the notification is obsolete, but that an implementor may wish to support that object to foster interoperability with older implementations.

\subsection{Mapping of the DESCRIPTION clause}

The DESCRIPTION clause, which must be present, contains a textual definition of the notification which provides all semantic definitions necessary for implementation, and should embody any information which would otherwise be communicated in any ASN.1 commentary annotations associated with the object. In particular, the DESCRIPTION clause should document which instances of the objects mentioned in the OBJECTS clause should be contained within notifications of this type.

\subsection{Mapping of the REFERENCE clause}

The REFERENCE clause, which need not be present, contains a textual cross-reference to a notification defined in some other information module. This is useful when de-osifying a 
MIB module produced by some other organization.

8.5. Mapping of the NOTIFICATION-TYPE value

The value of an invocation of the NOTIFICATION-TYPE macro is the name of the notification, which is an OBJECT IDENTIFIER, an administratively assigned name.

Sections 4.2 .6 and 4.2 .7 of [6] describe how the NOTIFICATION-TYPE macro is used to generate a SNMPV2-Trap-PDU or InformRequest-PDU, respectively. 


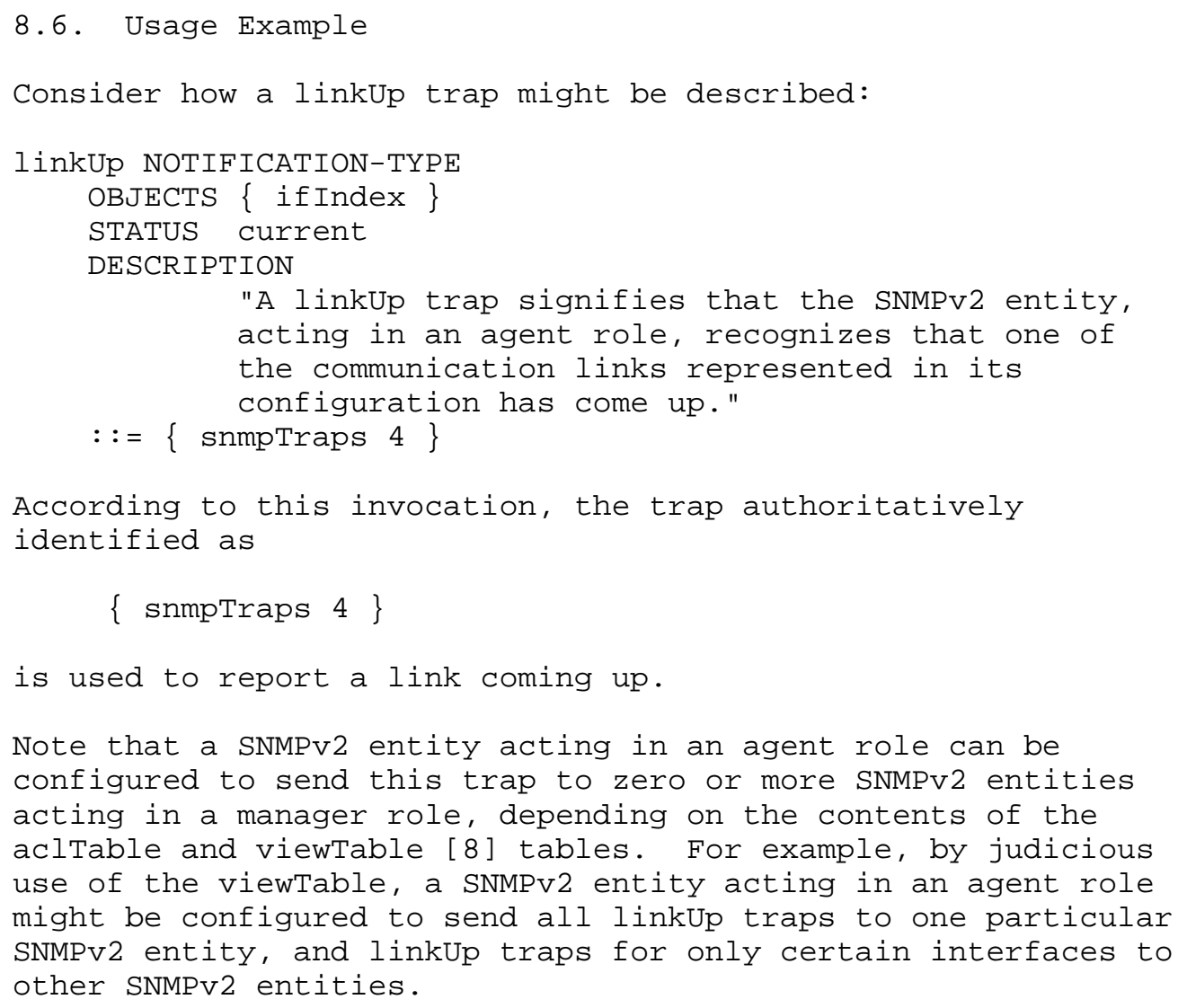




\section{Refined Syntax}

Some macros allow an object's syntax to be refined (e.g., the SYNTAX clause in the MODULE-COMPLIANCE macro [2]). However, not all refinements of syntax are appropriate. In particular, the object's primitive or application type must not be changed.

Further, the following restrictions apply:

\begin{tabular}{|c|c|c|c|c|}
\hline object syntax & $\begin{array}{l}\mathrm{R} \\
\text { range }\end{array}$ & $\begin{array}{l}\text { rictions to } \\
\text { enumeration }\end{array}$ & $\begin{array}{c}\text { Refinement } \\
\text { size }\end{array}$ & repertoire \\
\hline ----------------- & ----- & ----------- & ---- & ----------- \\
\hline INTEGER & (1) & (2) & - & - \\
\hline OCTET STRING & - & - & (3) & (4) \\
\hline OBJECT IDENTIFIER & - & - & - & - \\
\hline BIT STRING & - & (2) & - & - \\
\hline IpAddress & - & - & - & - \\
\hline Counter 32 & - & - & - & - \\
\hline Gauge 32 & (1) & - & - & - \\
\hline TimeTicks & - & - & - & - \\
\hline NsapAddress & - & - & - & - \\
\hline Counter 64 & - & - & - & - \\
\hline
\end{tabular}

where:

(1) the range of permitted values may be refined by raising the lower-bounds, by reducing the upper-bounds, and/or by reducing the alternative value/range choices;

(2) the enumeration of named-values may be refined by removing one or more named-values;

(3) the size in characters of the value may be refined by raising the lower-bounds, by reducing the upper-bounds, and/or by reducing the alternative size choices; or,

(4) the repertoire of characters in the value may be reduced by further sub-typing.

Otherwise no refinements are possible.

Note that when refining an object with a SYNTAX clause value of Integer32 or UInteger32, the refined SYNTAX is expressed as an INTEGER and the restrictions of the table above are used. 


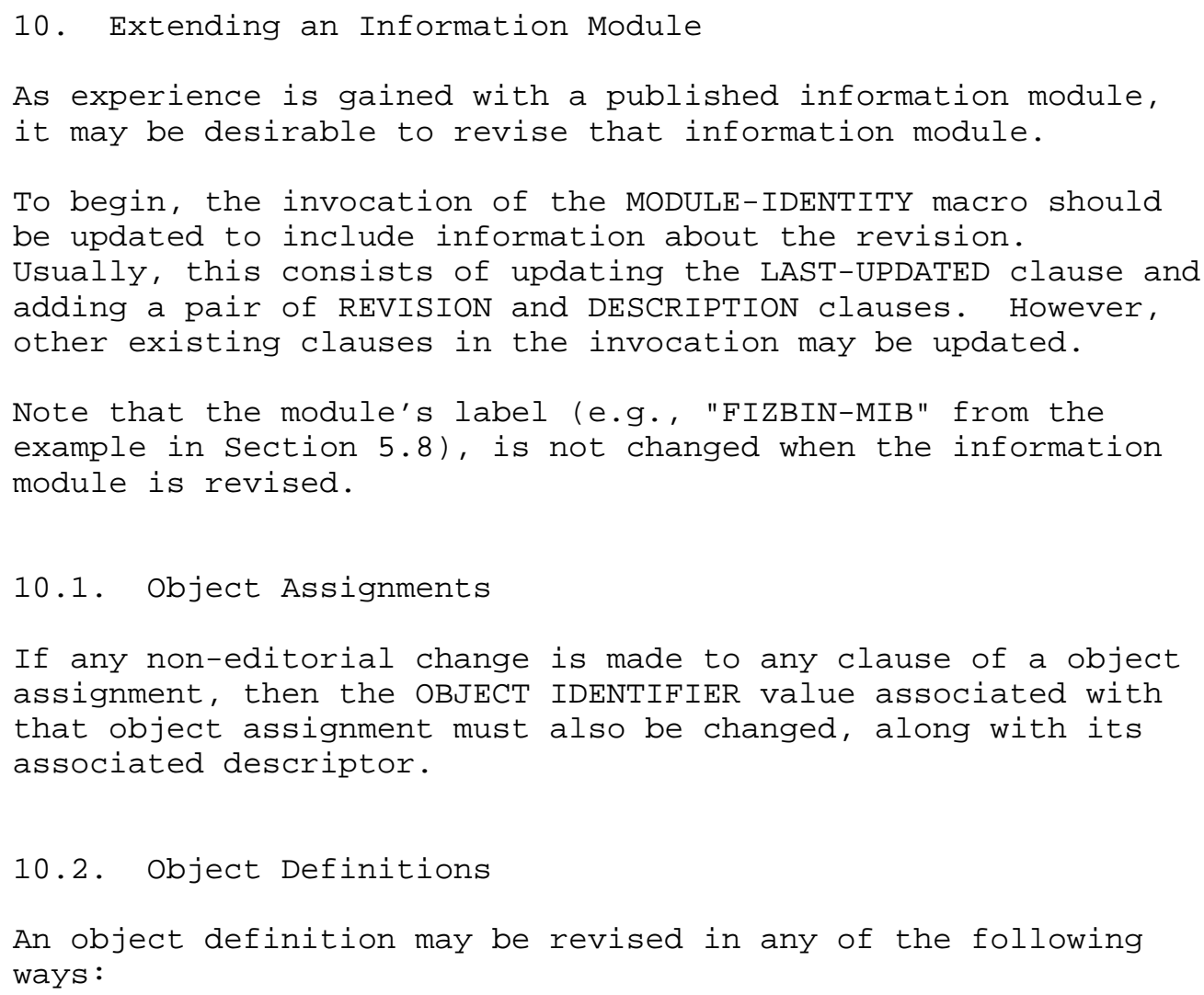

(1) A SYNTAX clause containing an enumerated INTEGER may have new enumerations added or existing labels changed.

(2) A STAtUs clause value of "current" may be revised as "deprecated" or "obsolete". Similarly, a STATUS clause value of "deprecated" may be revised as "obsolete".

(3) A DEFVAL clause may be added or updated.

(4) A REFERENCE clause may be added or updated.

(5) A UNITS clause may be added.

(6) A conceptual row may be augmented by adding new columnar objects at the end of the row. 
(7) Entirely new objects may be defined, named with previously unassigned OBJECT IDENTIFIER values.

Otherwise, if the semantics of any previously defined object are changed (i.e., if a non-editorial change is made to any clause other those specifically allowed above), then the OBJECT IDENTIFIER value associated with that object must also be changed.

Note that changing the descriptor associated with an existing object is considered a semantic change, as these strings may be used in an IMPORTS statement.

Finally, note that if an object has the value of its STATUS clause changed, then the value of its DESCRIPTION clause should be updated accordingly.

10.3. Notification Definitions

A notification definition may be revised in any of the following ways:

(1) A REFERENCE clause may be added or updated.

Otherwise, if the semantics of any previously defined notification are changed (i.e., if a non-editorial change is made to any clause other those specifically allowed above), then the OBJECT IDENTIFIER value associated with that notification must also be changed.

Note that changing the descriptor associated with an existing notification is considered a semantic change, as these strings may be used in an IMPORTS statement.

Finally, note that if an object has the value of its STATUS clause changed, then the value of its DESCRIPTION clause should be updated accordingly. 


\section{Appendix: de-OSIfying a MIB module}

There has been an increasing amount of work recently on taking MIBs defined by other organizations (e.g., the IEEE) and deosifying them for use with the Internet-standard network management framework. The steps to achieve this are straight-forward, though tedious. Of course, it is helpful to already be experienced in writing MIB modules for use with the Internet-standard network management framework.

The first step is to construct a skeletal MIB module, as shown earlier in section 5.8. The next step is to categorize the objects into groups. Optional objects are not permitted. Thus, when a MIB module is created, optional objects must be placed in a additional groups, which, if implemented, all objects in the group must be implemented. For the first pass, it is wisest to simply ignore any optional objects in the original MIB: experience shows it is better to define a core MIB module first, containing only essential objects; later, if experience demands, other objects can be added.

\subsection{Managed Object Mapping}

Next for each managed object class, determine whether there can exist multiple instances of that managed object class. If not, then for each of its attributes, use the OBJECT-TYPE macro to make an equivalent definition.

Otherwise, if multiple instances of the managed object class can exist, then define a conceptual table having conceptual rows each containing a columnar object for each of the managed object class's attributes. If the managed object class is contained within the containment tree of another managed object class, then the assignment of an object is normally required for each of the "distinguished attributes" of the containing managed object class. If they do not already exist within the MIB module, then they can be added via the definition of additional columnar objects in the conceptual row corresponding to the contained managed object class.

In defining a conceptual row, it is useful to consider the optimization of network management operations which will act upon its columnar objects. In particular, it is wisest to avoid defining more columnar objects within a conceptual row, 
than can fit in a single PDU. As a rule of thumb, a conceptual row should contain no more than approximately 20 objects. Similarly, or as a way to abide by the "20 object guideline", columnar objects should be grouped into tables according to the expected grouping of network management operations upon them. As such, the content of conceptual rows should reflect typical access scenarios, e.g., they should be organized along functional lines such as one row for statistics and another row for parameters, or along usage lines such as commonly-needed objects versus rarely-needed objects.

On the other hand, the definition of conceptual rows where the number of columnar objects used as indexes outnumbers the number used to hold information, should also be avoided. In particular, the splitting of a managed object class's attributes into many conceptual tables should not be used as a way to obtain the same degree of flexibility/complexity as is often found in MIBs with a myriad of optionals.

\subsubsection{Mapping to the SYNTAX clause}

When mapping to the SYNTAX clause of the OBJECT-type macro:

(1) An object with BOOLEAN syntax becomes a TruthValue [3].

(2) An object with INTEGER syntax becomes an Integer32.

(3) An object with ENUMERATED syntax becomes an INTEGER with enumerations, taking any of the values given which can be represented with an Integer 32 .

(4) An object with BIT STRING syntax but no enumerations becomes an OCTET STRING.

(5) An object with a character string syntax becomes either an OCTET STRING, or a Displaystring [3], depending on the repertoire of the character string.

(6) A non-tabular object with a complex syntax, such as REAL or EXTERNAL, must be decomposed, usually into an OCTET STRING (if sensible). As a rule, any object with a complicated syntax should be avoided. 
(7) Tabular objects must be decomposed into rows of columnar objects.

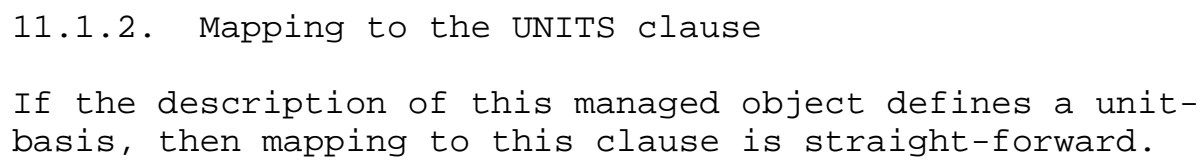




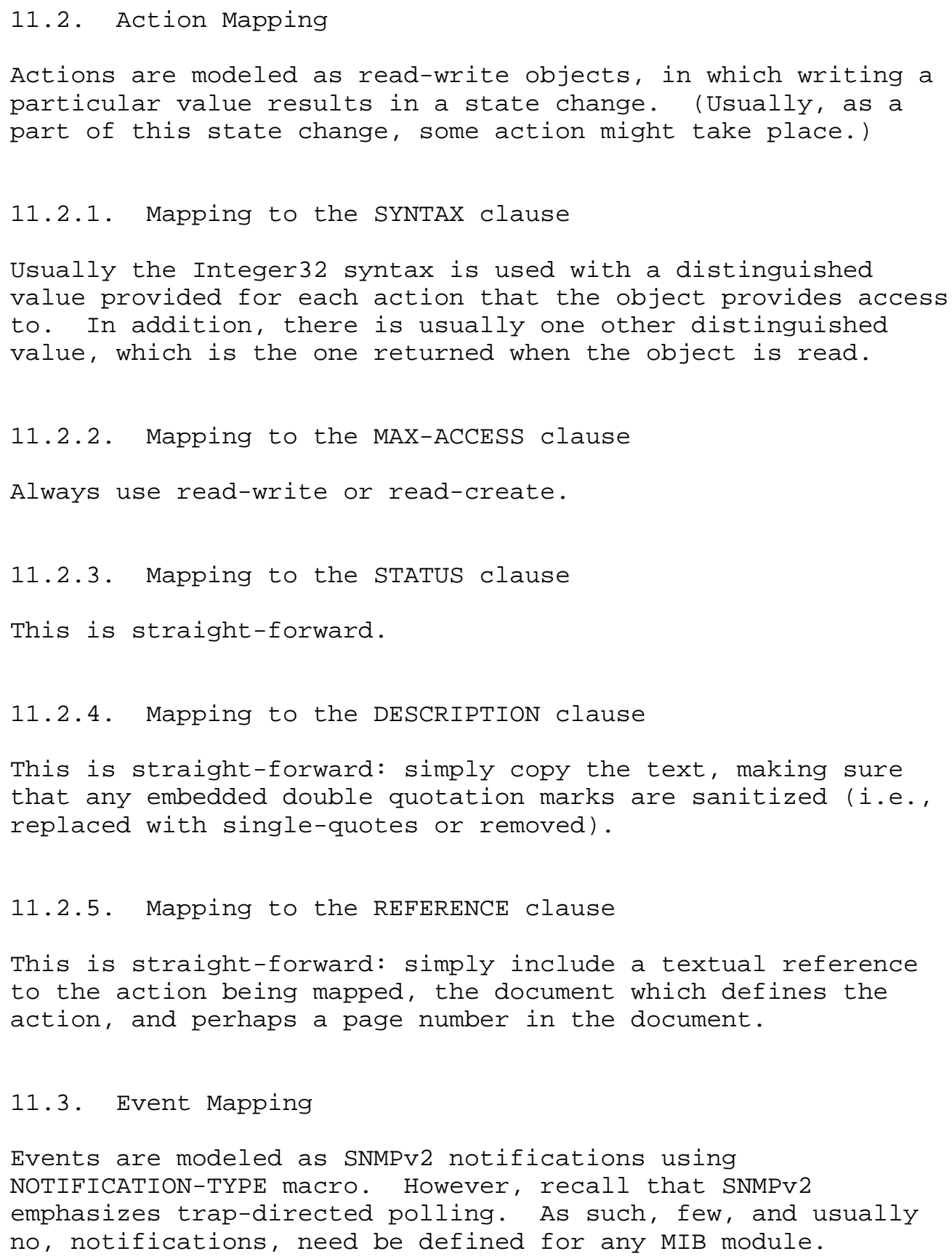


11.3.1. Mapping to the STATUS clause

This is straight-forward.

11.3.2. Mapping to the DESCRIPTION clause

This is straight-forward: simply copy the text, making sure that any embedded double quotation marks are sanitized (i.e., replaced with single-quotes or removed).

11.3.3. Mapping to the REFERENCE clause

This is straight-forward: simply include a textual reference to the notification being mapped, the document which defines the notification, and perhaps a page number in the document. 


\section{Acknowledgements}

The section on object definitions (and MIB de-osification) is based, in part, on RFCs 1155 and 1212. The IMPLIED keyword is based on a conversation with David T. Perkins in December, 1991.

The section on trap definitions is based, in part, on RFC 1215 .

Finally, the comments of the SNMP version 2 working group are gratefully acknowledged:

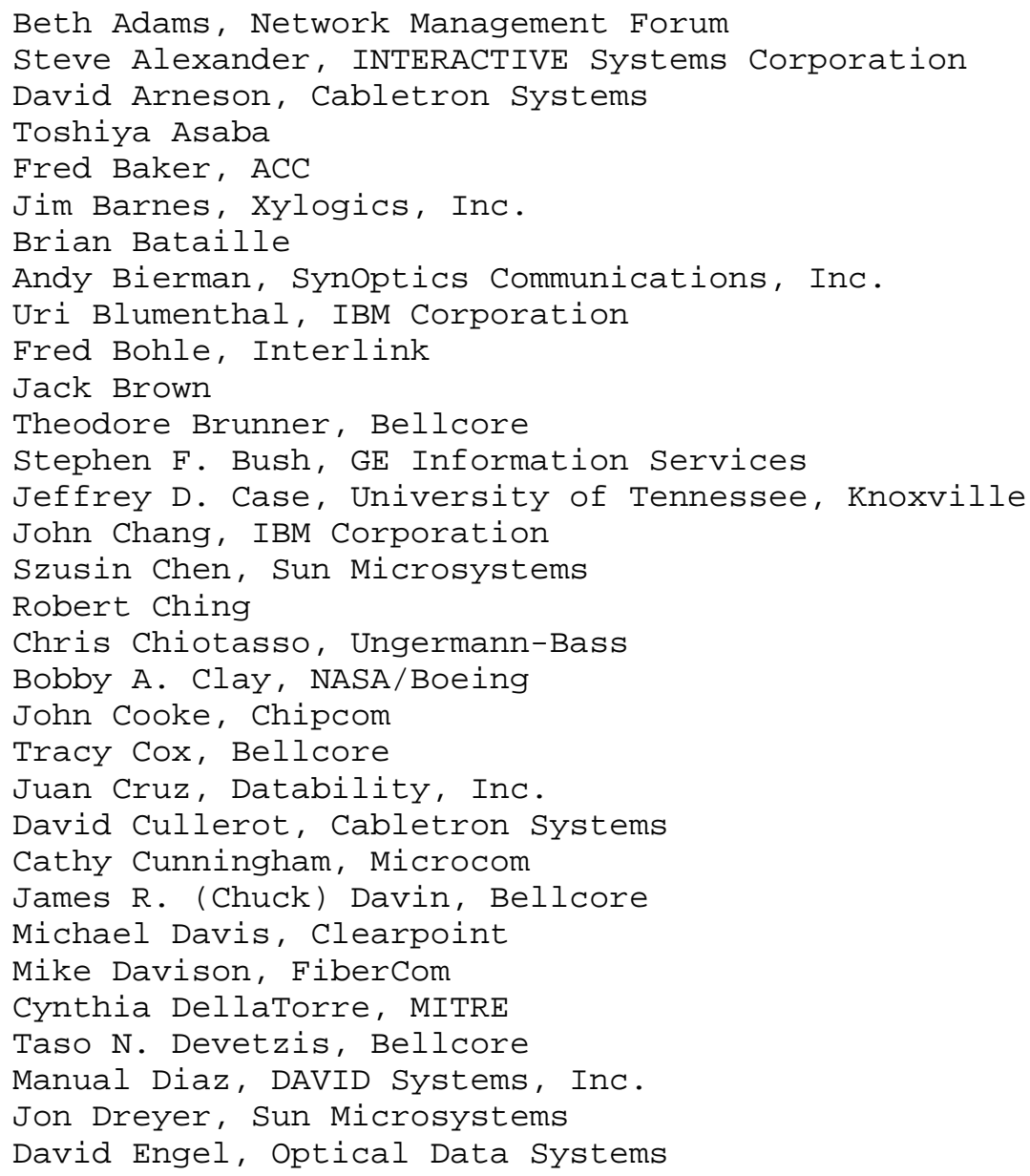




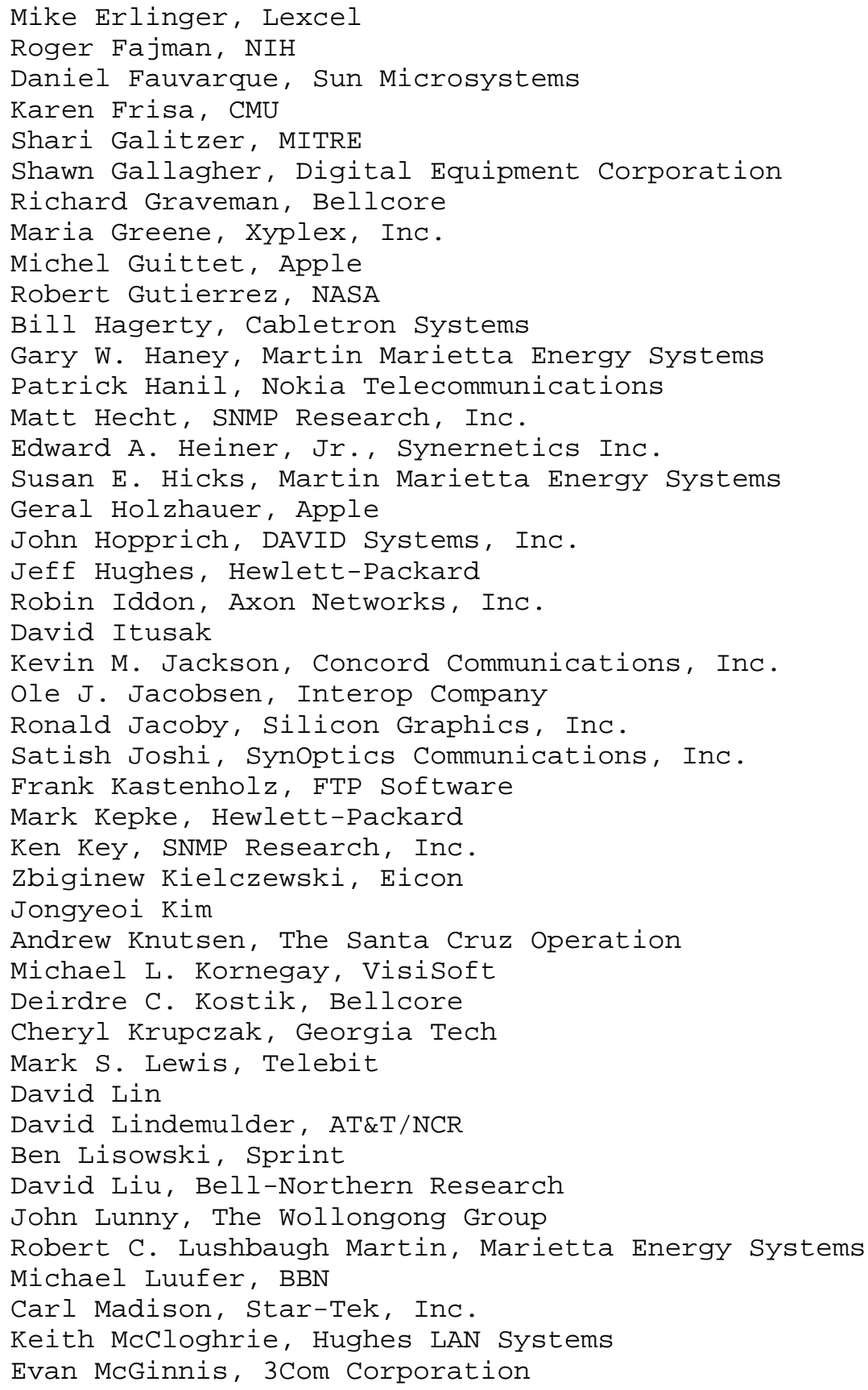




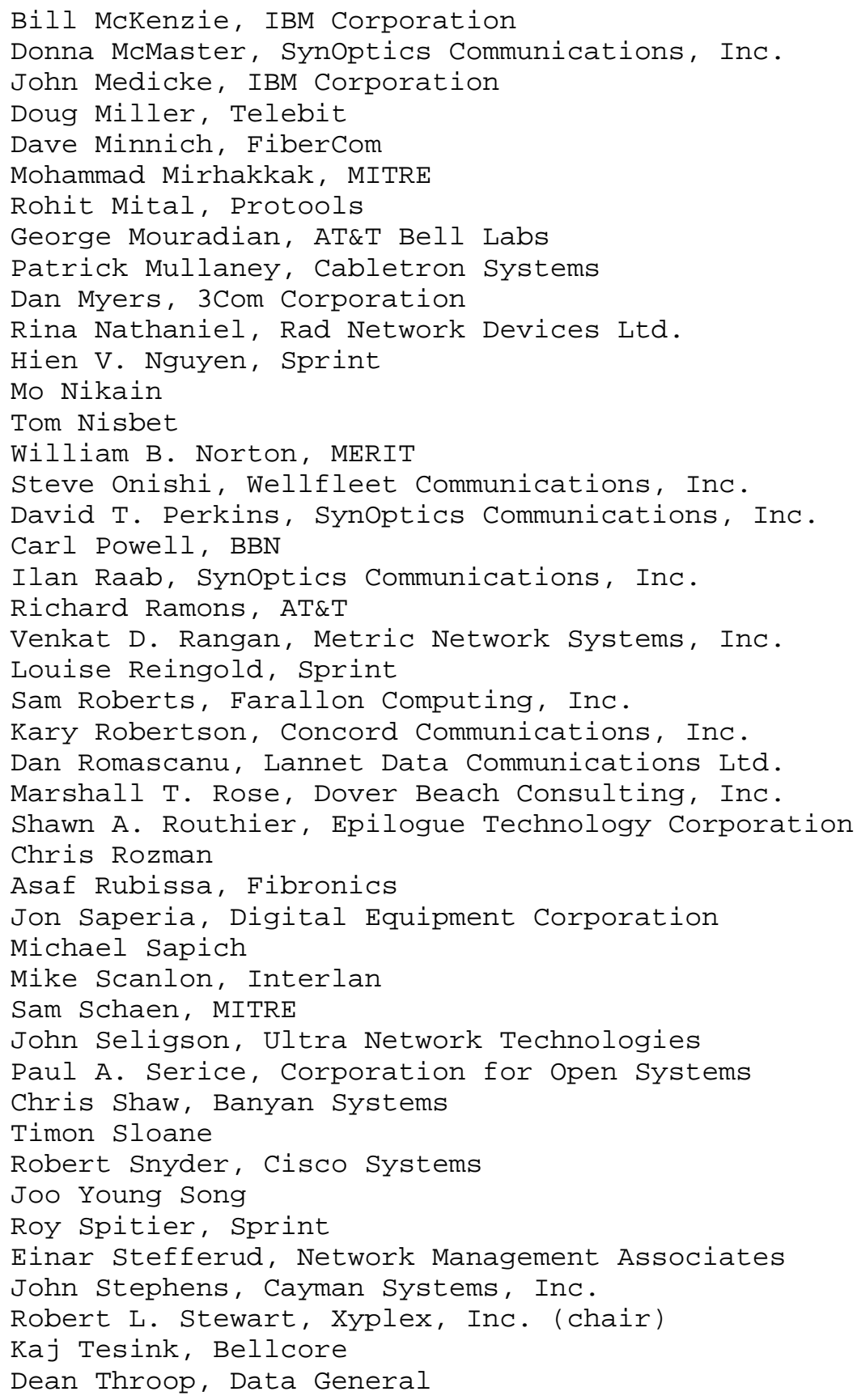




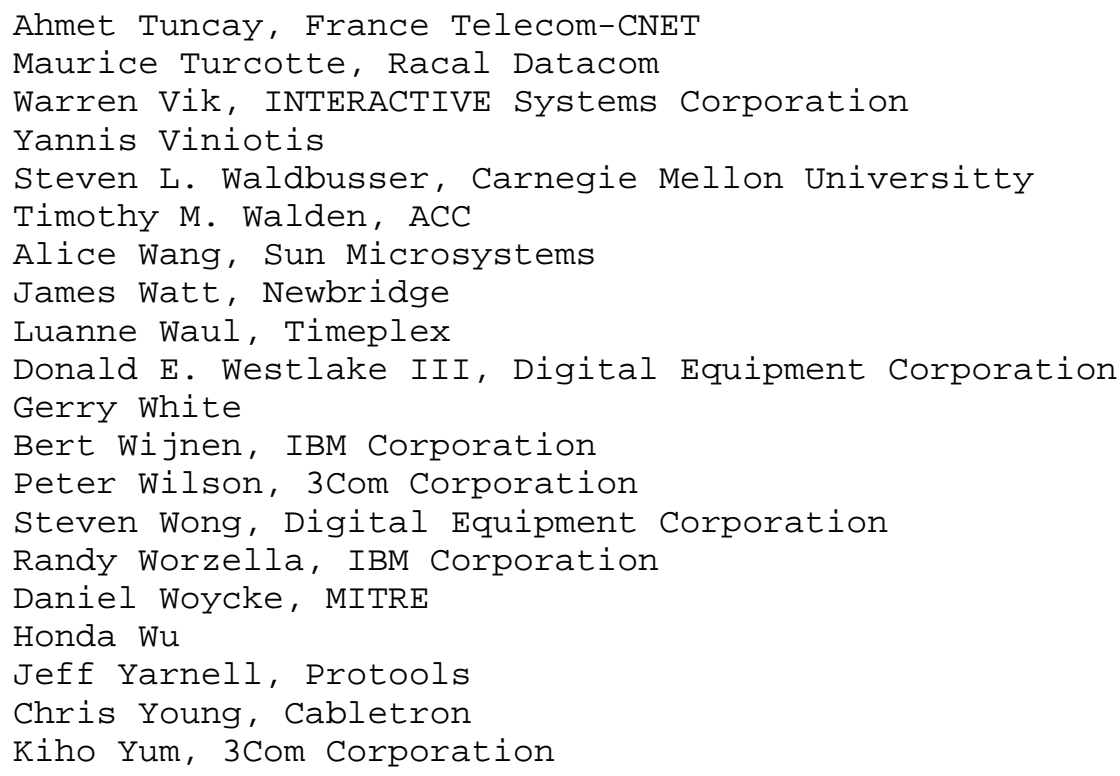




\section{References}

[1] Information processing systems - Open Systems Interconnection - Specification of Abstract Syntax Notation One (ASN.1), International Organization for Standardization. International Standard 8824, (December, 1987).

[2] Case, J., McCloghrie, K., Rose, M., and Waldbusser, S., "Conformance Statements for version 2 of the the Simple Network Management Protocol (SNMPv2)", RFC 1444, SNMP Research, Inc., Hughes LAN Systems, Dover Beach Consulting, Inc., Carnegie Mellon University, April 1993.

[3] Case, J., McCloghrie, K., Rose, M., and Waldbusser, S., "Textual Conventions for version 2 of the the Simple Network Management Protocol (SNMPv2)", RFC 1443, SNMP Research, Inc., Hughes LAN Systems, Dover Beach Consulting, Inc., Carnegie Mellon University, April 1993.

[4] Information processing systems - Open Systems Interconnection - Specification of Basic Encoding Rules for Abstract Syntax Notation one (ASN.1), International Organization for Standardization. International Standard 8825, (December, 1987).

[5] Case, J., McCloghrie, K., Rose, M., and Waldbusser, S., "Management Information Base for version 2 of the Simple Network Management Protocol (SNMPv2)", RFC 1450, SNMP Research, Inc., Hughes LAN Systems, Dover Beach Consulting, Inc., Carnegie Mellon University, April 1993.

[6] Case, J., McCloghrie, K., Rose, M., and Waldbusser, S., "Protocol Operations for version 2 of the Simple Network Management Protocol (SNMPv2)", RFC 1448, SNMP Research, Inc., Hughes LAN Systems, Dover Beach Consulting, Inc., Carnegie Mellon University, April 1993.

[7] McCloghrie, K., and Rose, M., "Management Information Base for Network Management of TCP/IP-based internets: MIB-II", STD 17, RFC 1213, March 1991.

[8] McCloghrie, K., and Galvin, J., "Party MIB for version 2 of the Simple Network Management Protocol (SNMPv2)", RFC 1447, Hughes LAN Systems, Trusted Information Systems, 
April 1993. 
14. Security Considerations

Security issues are not discussed in this memo.

15. Authors' Addresses

Jeffrey D. Case SNMP Research, Inc. 3001 Kimberlin Heights Rd. Knoxville, TN 37920-9716

US

Phone: +1 $615 \quad 573 \quad 1434$

Email: caselsnmp.com

Keith McCloghrie

Hughes LAN Systems

1225 Charleston Road

Mountain View, CA 94043

US

Phone: +1 4159667934

Email: kzmehls.com

Marshall T. Rose

Dover Beach Consulting, Inc.

420 Whisman court

Mountain View, CA 94043-2186

US

Phone: +1415968 1052

Email: mroseddbc.mtview.ca.us

Steven Waldbusser

Carnegie Mellon University

4910 Forbes Ave

Pittsburgh, PA 15213

US

Phone: +1 4122686628

Email: waldbusserecmu.edu 